\title{
SYSTEMS WITH HYSTERESIS IN THE FEEDBACK LOOP: EXISTENCE, REGULARITY AND ASYMPTOTIC BEHAVIOUR OF SOLUTIONS*
}

\author{
Hartmut Logemann ${ }^{1}$ And Eugene P. Ryan ${ }^{1}$
}

\begin{abstract}
An existence and regularity theorem is proved for integral equations of convolution type which contain hysteresis nonlinearities. On the basis of this result, frequency-domain stability criteria are derived for feedback systems with a linear infinite-dimensional system in the forward path and a hysteresis nonlinearity in the feedback path. These stability criteria are reminiscent of the classical circle criterion which applies to static sector-bounded nonlinearities. The class of hysteresis operators under consideration contains many standard hysteresis nonlinearities which are important in control engineering such as backlash (or play), plastic-elastic (or stop) and Prandtl operators. Whilst the main results are developed in the context of integral equations of convolution type, applications to well-posed state space systems are also considered.
\end{abstract}

Mathematics Subject Classification. 45M05, 45M10, 47J40, 93C10, 93C25, 93D05, 93D10, $93 \mathrm{D} 25$.

Received June 12, 2002. Revised November 12, 2002.

\section{INTRODUCTION}

Consider the feedback system shown in Figure 1, where $G$ is a convolution operator and $r_{1}$ and $r_{2}$ are realvalued input and disturbance signals, respectively. In the special case of static nonlinearities $\Phi=\varphi$, if the feedback system is stable for every locally Lipschitz $\varphi: \mathbb{R} \rightarrow \mathbb{R}$ satisfying the sector condition

$$
a w^{2} \leq \varphi(w) w \leq b w^{2}, \quad \forall w \in \mathbb{R},
$$

then the system is said to be absolutely stable (with respect to the sector determined by $a$ and $b$ ). There is a rich literature on absolute stability theory: see, for example, Curtain et al. [4], Gripenberg et al. [6], Hahn [7], Vidyasagar [19] and the references therein. In the context of static nonlinearities, the circle criterion and the Popov criterion are two fundamentally important criteria for absolute stability. In the present paper we develop absolute-stability-type criteria for the system shown in Figure 1 in cases wherein $\Phi$ belongs to a certain class of hysteresis nonlinearities. A substantial literature on the mathematical theory of hysteresis phenomena exists, see for example Brokate [1], Brokate and Sprekels [2]: of particular importance in a systems and control context is the pioneering work of Krasnosel'skiı̌ and Pokrovskiı̌ [8]. A hysteresis nonlinearity is simply a causal and

Keywords and phrases: Absolute stability, asymptotic behaviour, frequency-domain stability criteria, hysteresis,

infinite-dimensional systems, integral equations, regularity of solutions.

* This work was supported by UK EPSRC Council (Grant GR/L78086).

${ }^{1}$ Department of Mathematical Sciences, University of Bath, Bath BA2 7AY, U.K.;

e-mail: hl@maths.bath.ac.uk, epr@maths.bath.ac.uk 


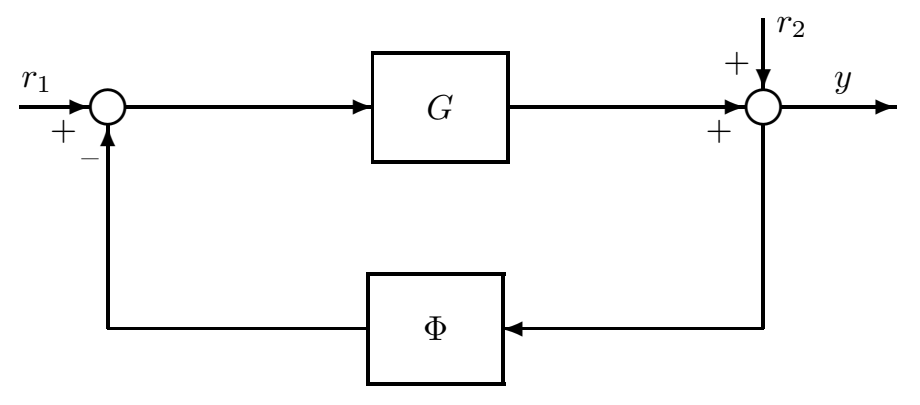

FiguRE 1. Feedback system with nonlinearity.

rate-independent operator mapping the space of continuous functions defined on $\mathbb{R}_{+}:=[0, \infty)$ into itself. In Section 3, we introduce a class of hysteresis operators which contain many standard hysteresis nonlinearities such as backlash (or play), plastic-elastic (or stop) and Prandtl operators. Our treatment of hysteresis operators in Section 3 has been strongly influenced by Chapter 2 in [2].

To provide a framework for the later stability analysis, we first investigate, in Section 2, existence, uniqueness and regularity of solutions of the integral equation

$$
u(t)=r(t)-\int_{0}^{t} g(t-s)(\Phi(u))(s) \mathrm{d} s
$$

where we assume that $g$ is locally integrable, $\Phi: C\left(\mathbb{R}_{+}\right) \rightarrow C\left(\mathbb{R}_{+}\right)$is a causal operator and $r$ is continuous. We show that (2) has a unique maximal continuous solution provided that $\Phi$ satisfies a local Lipschitz-type condition in the space of continuous functions. Under the assumptions that $\Phi$ satisfies a local Lipschitz-type condition in the Sobolev space $W^{1,1}\left(\mathbb{R}_{+}\right)$and $r$ is locally absolutely continuous, we show that $(2)$ has a unique maximal locally absolutely continuous solution.

In Section 4, we consider the feedback system shown in Figure 1, where $G$ is a convolution operator mapping $L^{2}\left(\mathbb{R}_{+}\right)$boundedly into $L^{2}\left(\mathbb{R}_{+}\right), \Phi$ is a hysteresis operator, $r_{1}$ and $r_{2}$ are input and disturbance signals, respectively. Assuming that $G$ has a convolution kernel $g$ in $L^{1}\left(\mathbb{R}_{+}\right)$, the feedback system in Figure 1 translates to the integral equation

$$
y(t)=\int_{0}^{t} g(t-s) r_{1}(s) \mathrm{d} s+r_{2}(t)-\int_{0}^{t} g(t-s)(\Phi(y)(s) \mathrm{d} s
$$

which is of the form (2). If certain natural conditions on $g, \Phi, r_{1}$ and $r_{2}$ are satisfied, we show that the solution $y$ exists on $\mathbb{R}_{+}$(no finite escape-time), $y$ and $\Phi(y)$ are bounded and $y(t)$ and $(\Phi(y))(t)$ converge to finite limits as $t \rightarrow \infty$, provided that

$$
\inf _{\omega \in \mathbb{R}} \operatorname{Re} \mathbf{G}(i \omega)>-1 / \lambda
$$

where $\mathbf{G}$ denotes the Laplace transform of $g$ and $\lambda>0$ is a Lipschitz-type constant associated with $\Phi$. Moreover, we give estimates of the supremum norms of $y$ and $\Phi(y)$ in terms of the signals $r_{1}$ and $r_{2}$. We prove a similar result for the feedback system obtained if, in Figure 1, $G$ is replaced by the operator $H$ given by $(H v)(t)=\int_{0}^{t}(G v)(s) \mathrm{d} s$ (that is, if an integrator is introduced into the forward path), a situation which is of major importance in control theory. Note that the operator $H$ is usually "unstable" in the sense that it does not map $L^{2}\left(\mathbb{R}_{+}\right)$boundedly into $L^{2}\left(\mathbb{R}_{+}\right)$. We mention that (3) is reminiscent of the frequency-domain condition posited in the classical circle criterion (in the context of feedback systems with exponentially stable linear system and static nonlinearity satisfying (1) with $a=0)$. 
In Section 5, we apply the results in Section 4 to the case where the convolution operator $G$ is realized by a well-posed linear infinite-dimensional state-space system. The class of well-posed state-space systems is widely documented in the literature, see for example $[5,14,15,20]$. We remark that the class of well-posed, linear, infinite-dimensional systems is rather general: it includes most distributed parameter systems and all time-delay systems (retarded and neutral) which are of interest in applications. We emphasize that the results in Sections 4 and 5 are new even if the underlying linear system is finite-dimensional.

Notation and terminology. Let $I \subset \mathbb{R}_{+}$be an interval, where $\mathbb{R}_{+}:=[0, \infty)$. The space of continuous functions $I \rightarrow \mathbb{R}$ is denoted by $C(I)$. If $I$ is compact, then, endowed with the supremum norm $\|v\|_{C(I)}=$ $\sup _{t \in I}|v(t)|, C(I)$ is a Banach space. For $\alpha \geq 0$, let $W^{1,1}([0, \alpha])$ denote the Sobolev space of absolutely continuous functions $v:[0, \alpha] \rightarrow \mathbb{R}$, equipped with the norm $\|v\|_{W^{1,1}([0, \alpha])}=|v(0)|+\int_{0}^{\alpha}\left|v^{\prime}(t)\right| \mathrm{d} t$. This norm is readily shown to be equivalent to the usual norm on $W^{1,1}([0, \alpha])$ given by $\|v\|_{1,1}=\|v\|_{1}+\left\|v^{\prime}\right\|_{1}$ (where $\|\cdot\|_{1}$ denotes the $L^{1}$ norm). Note that $\|v\|_{W^{1,1}([0, \alpha])}$ is equal to the total variation of the function $v$. For $0<\beta \leq \infty$, we denote, by $W_{\text {loc }}^{1,1}([0, \beta))$, the space of locally absolutely continuous functions $v:[0, \beta) \rightarrow \mathbb{R}$, that is $v \in$ $W_{\text {loc }}^{1,1}([0, \beta))$ if and only if $\left.v\right|_{[0, \alpha]} \in W^{1,1}([0, \alpha])$ for all $0 \leq \alpha<\beta$.

For $\alpha=0, C([0, \alpha])=W^{1,1}([0, \alpha]):=\mathbb{R}$. Let $\gamma, \delta>0$. For $w \in C([0, \alpha])$ (with $\left.\alpha \geq 0\right)$, let $w_{\gamma}$ denote the function in $C([0, \alpha+\gamma])$ given by

$$
t \mapsto w_{\gamma}(t):= \begin{cases}w(t), & t \in[0, \alpha] \\ w(\alpha), & t \in(\alpha, \alpha+\gamma]\end{cases}
$$

Obviously, if $w \in W^{1,1}([0, \alpha])$, then $w_{\gamma} \in W^{1,1}([0, \alpha+\gamma])$. We define

$$
\begin{aligned}
\mathcal{C}(w ; \delta, \gamma) & :=\left\{v \in C([0, \alpha+\gamma]):\left.v\right|_{[0, \alpha]}=w,\left\|v-w_{\gamma}\right\|_{C([0, \alpha+\gamma])} \leq \delta\right\} \\
& =\left\{v \in C([0, \alpha+\gamma]):\left.v\right|_{[0, \alpha]}=w, \sup _{t \in[\alpha, \alpha+\gamma]}|v(t)-w(\alpha)| \leq \delta\right\}
\end{aligned}
$$

and

$$
\begin{aligned}
\mathcal{W}(w ; \delta, \gamma) & :=\left\{v \in W^{1,1}([0, \alpha+\gamma]):\left.v\right|_{[0, \alpha]}=w,\left\|v-w_{\gamma}\right\|_{W^{1,1}([0, \alpha+\gamma])} \leq \delta\right\} \\
& =\left\{v \in W^{1,1}([0, \alpha+\gamma]):\left.v\right|_{[0, \alpha]}=w, \int_{\alpha}^{\alpha+\gamma}\left|v^{\prime}(t)\right| \mathrm{d} t \leq \delta\right\} .
\end{aligned}
$$

Equipped with the metric

$$
\left(v_{1}, v_{2}\right) \mapsto\left\|v_{1}-v_{2}\right\|
$$

where $\|\cdot\|$ denotes the norm on $C([0, \alpha+\gamma])$ or on $W^{1,1}([0, \alpha+\gamma])$ as appropriate, $\mathcal{C}(w ; \delta, \gamma)$ and $\mathcal{W}(w ; \delta, \gamma)$ are complete metric spaces.

Let $Z$ be a Banach space and let $\beta \in \mathbb{R}$. We define the exponentially weighted $L^{p}$-space $L_{\beta}^{p}\left(\mathbb{R}_{+}, Z\right):=$ $\left\{f: f(\cdot) \exp (-\beta \cdot) \in L^{p}\left(\mathbb{R}_{+}, Z\right)\right\}$ and endow it with the norm $\|f\|_{L_{\beta}^{p}\left(\mathbb{R}_{+}, Z\right)}:=\left(\int_{0}^{\infty}\left\|\mathrm{e}^{-\beta t} f(t)\right\|^{p} \mathrm{~d} t\right)^{1 / p}$. Setting $\mathbb{C}_{\beta}:=\{s \in \mathbb{C}: \operatorname{Re} s>\beta\}$, let $H^{\infty}\left(\mathbb{C}_{\beta}, Z\right)$ denote the space of bounded holomorphic $Z$-valued functions defined on $\mathbb{C}_{\beta}$ and let $H^{2}\left(\mathbb{C}_{\beta}, Z\right)$ denote the usual Hardy-Lebesgue space of square-integrable $Z$-valued functions defined on $\mathbb{C}_{\beta}$. For simplicity, we write $L_{\beta}^{p}\left(\mathbb{R}_{+}\right)$in place of $L_{\beta}^{p}\left(\mathbb{R}_{+}, \mathbb{R}\right), H^{\infty}\left(\mathbb{C}_{\beta}\right)$ in place of $H^{\infty}\left(\mathbb{C}_{\beta}, \mathbb{C}_{)}\right.$and $H^{2}\left(\mathbb{C}_{\beta}\right)$ in place of $H^{2}\left(\mathbb{C}_{\beta}, \mathbb{C}\right)$.

The space of bounded linear operators from a Banach space $Z_{1}$ to a Banach space $Z_{2}$ is denoted by $\mathcal{L}\left(Z_{1}, Z_{2}\right)$; we write $\mathcal{L}(Z)$ in place of $\mathcal{L}(Z, Z)$. Let $A$ : $\operatorname{dom}(A) \subset Z \rightarrow Z$ be a linear operator, where $\operatorname{dom}(A)$ denotes the domain of $A$; the resolvent set of $A$ is denoted by $\varrho(A)$. Finally, an operator $\Phi: C\left(\mathbb{R}_{+}\right) \rightarrow C\left(\mathbb{R}_{+}\right)$is causal if, for $v_{1}, v_{2} \in C\left(\mathbb{R}_{+}\right)$with $v_{1}(t)=v_{2}(t)$ for all $t \in[0, \alpha]$, it follows that $\left(\Phi\left(v_{1}\right)\right)(t)=\left(\Phi\left(v_{2}\right)\right)(t)$ for all $t \in[0, \alpha]$. 


\section{A Class of integral EQUations}

Let $\Phi: C\left(\mathbb{R}_{+}\right) \rightarrow C\left(\mathbb{R}_{+}\right)$be a causal operator, $g \in L_{\text {loc }}^{1}\left(\mathbb{R}_{+}\right)$and $r \in C\left(\mathbb{R}_{+}\right)$. We consider integral equations of the form

$$
u(t)=r(t)-\int_{0}^{t} g(t-s)(\Phi(u))(s) \mathrm{d} s
$$

which we also write concisely as

$$
u=r-g \star \Phi(u) .
$$

In the following, let $I$ be an interval of the form $I=[0, \alpha]$ (with $0 \leq \alpha<\infty)$ or $I=[0, \beta)($ with $0<\beta \leq \infty)$. In order to define the concept of a (local) solution of (5), we need to give a meaning to $\Phi(v)$ if $v \in C(I)$ (recall that $\Phi$ acts on continuous function defined on the half-line $\left.\mathbb{R}_{+}\right)$. For $v \in C([0, \alpha])$, we interpret $\Phi(v)$ as the element of $C([0, \alpha])$ given by $(\Phi(\tilde{v}))(t)$ for all $t \in[0, \alpha]$, where $\tilde{v}$ is any continuous extension of $v$ to $\mathbb{R}_{+}$. By causality of $\Phi$, the function $\Phi(v) \in C([0, \alpha])$ is invariant with respect to the choice of the extension $\tilde{v}$. For a continuous function $v:[0, \beta) \rightarrow \mathbb{R}(0<\beta<\infty)$, we interpret $\Phi(v)$ as the continuous function $[0, \beta) \rightarrow \mathbb{R}$ with the property that $(\Phi(v))(t)=\left(\Phi\left(\left.v\right|_{[0, \alpha]}\right)\right)(t)$ for all $t \in[0, \alpha]$ and for all $\alpha \in(0, \beta)$. Causality of $\Phi$ guarantees that $\Phi(v)$ is well-defined.

By a solution of (5) on an interval $I$ we mean a function $u \in C(I)$ that satisfies (5) for all $t \in I$. A solution $u \in C(I)$ is maximal, if $u$ has no proper right extension that is also a solution. A function $u \in C(I)$ is called a maximal locally absolutely continuous solution, if $u$ is a is a locally absolutely continuous solution and there does not exist a locally absolutely continuous proper right extension that is also a solution.

Our goal is to establish existence and uniqueness of solutions of (5). To this end, we introduce the following local Lipschitz-type assumptions:

(LC) for all $\alpha \geq 0$ and all $w \in C([0, \alpha])$, there exist constants $\lambda, \delta, \gamma>0$ such that

$$
\left\|\Phi\left(v_{1}\right)-\Phi\left(v_{2}\right)\right\|_{C([0, \alpha+\gamma])} \leq \lambda\left\|v_{1}-v_{2}\right\|_{C([0, \alpha+\gamma])} \quad \forall v_{1}, v_{2} \in \mathcal{C}(w ; \delta, \gamma) ;
$$

$(\mathbf{L W}) \Phi\left(W_{\text {loc }}^{1,1}\left(\mathbb{R}_{+}\right)\right) \subset W_{\text {loc }}^{1,1}\left(\mathbb{R}_{+}\right)$and, for all $\alpha \geq 0$ and all $w \in W^{1,1}([0, \alpha])$, there exist constants $\lambda, \delta, \gamma>0$ such that

$$
\left\|\Phi\left(v_{1}\right)-\Phi\left(v_{2}\right)\right\|_{W^{1,1}([0, \alpha+\gamma])} \leq \lambda\left\|v_{1}-v_{2}\right\|_{W^{1,1}([0, \alpha+\gamma])} \quad \forall v_{1}, v_{2} \in \mathcal{W}(w ; \delta, \gamma) .
$$

We first present a technical result which underpins the subsequent existence and uniqueness theorem.

Lemma 2.1. Let $\Phi: C\left(\mathbb{R}_{+}\right) \rightarrow C\left(\mathbb{R}_{+}\right)$be causal, $g \in L_{\mathrm{loc}}^{1}\left(\mathbb{R}_{+}\right), r \in C\left(\mathbb{R}_{+}\right), \alpha \geq 0$ and $w \in C([0, \alpha])$ with $w(\alpha)=(r-g \star(\Phi(w)))(\alpha)$. Let $\Gamma_{\eta}$, parameterized by $\eta>0$, denote the operator defined on $C([0, \alpha+\eta])$ by

$$
\left(\Gamma_{\eta}(x)\right)(t):= \begin{cases}w(t), & t \in[0, \alpha] \\ (r-g \star(\Phi(x)))(t), & t \in(\alpha, \alpha+\eta] .\end{cases}
$$

The following statements hold.

(a) If (LC) is satisfied, then there exists $\delta>0$ such that, for all $\eta>0$ sufficiently small, $\Gamma_{\eta}(\mathcal{C}(w ; \delta, \eta))$ $\subset \mathcal{C}(w ; \delta, \eta)$ and $\Gamma_{\eta}$ is a strict contraction on $\mathcal{C}(w ; \delta, \eta)$.

(b) Let $r \in W_{\text {loc }}^{1,1}\left(\mathbb{R}_{+}\right)$and $w \in W^{1,1}([0, \alpha])$. If (LW) is satisfied, then there exists $\delta>0$ such that, for all $\eta>0$ sufficiently small, $\Gamma_{\eta}(\mathcal{W}(w ; \delta, \eta)) \subset \mathcal{W}(w ; \delta, \eta)$ and $\Gamma_{\eta}$ is a strict contraction on $\mathcal{W}(w ; \delta, \eta)$.

The proof of the above lemma can be found in the Appendix. 
The following existence and uniqueness theorem is the main result of this section.

Theorem 2.2. Let $g \in L_{\text {loc }}^{1}\left(\mathbb{R}_{+}\right), r \in C\left(\mathbb{R}_{+}\right)$and let $\Phi: C\left(\mathbb{R}_{+}\right) \rightarrow C\left(\mathbb{R}_{+}\right)$be causal.

(a) Assume that $\Phi$ satisfies (LC). Then equation (5) has a unique maximal solution $u \in C\left(\left[0, t^{*}\right)\right)$, where $0<$ $t^{*} \leq \infty$. If $t^{*}<\infty$, then $\lim \sup _{t \uparrow t^{*}}|(\Phi(u))(t)|=\infty$. If $r \in W_{\mathrm{loc}}^{1,1}\left(\mathbb{R}_{+}\right)$and $g$ is locally of bounded variation, then the maximal solution $u$ is locally absolutely continuous, i.e., $u \in W_{\mathrm{loc}}^{1,1}\left(\left[0, t^{*}\right)\right)$.

(b) Assume that $\Phi$ satisfies (LW) and $r \in W_{\text {loc }}^{1,1}\left(\mathbb{R}_{+}\right)$. Then equation (5) has a unique maximal locally absolutely continuous solution $u:\left[0, t^{*}\right) \rightarrow \mathbb{R}$. If $t^{*}<\infty$, then $\int_{0}^{t}\left|u^{\prime}(s)\right| \mathrm{d} s \rightarrow \infty$ as $t \uparrow t^{*}$.

Proof. (a) We proceed in several steps.

Step 1: Extending solutions defined on compact intervals.

Assume $\alpha \geq 0$ and $w \in C([0, \alpha])$ is a solution of (5). By Lemma 2.1, there exists $\delta>0$ such that, for all sufficiently small $\eta>0, \Gamma_{\eta}: \mathcal{C}(w ; \delta, \eta) \rightarrow \mathcal{C}(w ; \delta, \eta)$ is a strict contraction and so has a unique fixed point $u$. By definition of $\Gamma_{\eta},\left.u\right|_{[0, \alpha]}=w$ and $u(t)=\left(\Gamma_{\eta}(u)\right)(t)=(r-g \star(\Phi(u))(t)$ for all $t \in(\alpha, \alpha+\eta])$. Therefore, the function $u$ is is a proper right extension of $w$ and $u$ is a solution of (5) on $[0, \alpha+\eta]$. Moreover, if $v$ is any other proper right extension of $w$ solving (5), then $v \in \mathcal{C}(w ; \delta, \eta)$ for sufficiently small $\eta>0$. It follows from the uniqueness of $u$ in $\mathcal{C}(w ; \delta, \eta)$ that $u=v$ on $[0, \alpha+\eta]$, provided that $\eta>0$ is sufficiently small.

Step 2: Extended uniqueness.

Let $\alpha_{1}, \alpha_{2}>0$ and let $u_{1} \in C\left(\left[0, \alpha_{1}\right)\right)$ and $u_{2} \in C\left(\left[0, \alpha_{2}\right)\right)$ be solutions. Set $\beta:=\min \left\{\alpha_{1}, \alpha_{2}\right\}$. We claim that $u_{1}=u_{2}$ on $[0, \beta)$. Seeking a contradiction, suppose that the claim is false. Then

$$
\alpha^{*}:=\inf \left\{t \in[0, \beta): u_{1}(t) \neq u_{2}(t)\right\}<\beta
$$

By the uniqueness property at the end of Step 1 (applied to the case $\alpha=0$ and $w=r(0)$ ), $\alpha^{*}>0$ and we define $w(t):=u_{1}(t)=u_{2}(t)$ for all $t \in\left[0, \alpha^{*}\right]$. Therefore, $w \in C\left(\left[0, \alpha^{*}\right]\right)$ is a solution of $(5)$ on $\left[0, \alpha^{*}\right]$ and so, again by Step 1 , there exists $\eta>0$ and a solution $u \in C\left(\left[0, \alpha^{*}+\eta\right]\right)$ with $u_{1}(t)=u_{2}(t)=u(t)$ on $\left[0, \alpha^{*}+\eta\right]$. This contradicts the definition of $\alpha^{*}$.

Step 3: Existence of a maximal solution.

Let $T$ be the set of all $\tau>0$ such that there exists a solution $u^{\tau}$ of (5) on the interval [0, $\left.\tau\right]$. By Step 1 (applied to the case $\alpha=0$ and $w=r(0)), T \neq \emptyset$. Let $t^{*}:=\sup T$ and define a function $u:\left[0, t^{*}\right) \rightarrow \mathbb{R}$ by setting

$$
u(t)=u^{\tau}(t), \quad \text { for } t \in[0, \tau), \text { where } \tau \in T
$$

By Step 2 the function $u$ is well-defined, i.e., the definition of $u(t)$ for a particular value $t \in\left[0, t^{*}\right)$ does not depend on the choice of $\tau \in T \cap(t, \infty)$. Moreover, it is clear that $u$ is a maximal solution of (5). Uniqueness of this maximal solution follows from Step 2.

Step 4: Unboundedness of $\Phi(u)$ if $t^{*}<\infty$.

Assume that $t^{*}<\infty$. Seeking a contradiction, suppose that $\Phi(u)$ is bounded. Define $\varphi \in L^{\infty}\left(\left[0, t^{*}\right]\right)$ by $\left.\varphi\right|_{\left[0, t^{*}\right)}=\Phi(u)$ and $\varphi\left(t^{*}\right)=0$. Then $t \mapsto(g \star \varphi)(t)$ is continuous on $\left[0, t^{*}\right]$ and $u(t)=(r-g \star \varphi)(t)$ for all $t \in\left[0, t^{*}\right)$. Therefore, $l:=(r-g \star \varphi)\left(t^{*}\right)=\lim _{t \uparrow t^{*}} u(t)$, and so $\tilde{u} \in C\left(\left[0, t^{*}\right]\right)$ given by $\left.\tilde{u}\right|_{\left[0, t^{*}\right)}=u$ and $\tilde{u}\left(t^{*}\right)=l$ is an extension of $u$ and is a solution of (5). This, together with Step 1, contradicts maximality of $u$.

Step 5: Absolute continuity.

Assume that $r \in W_{\text {loc }}^{1,1}\left(\mathbb{R}_{+}\right)$and $g$ is locally of bounded variation. Let $u \in C\left(\left[0, t^{*}\right)\right)$ be the unique maximal solution. Then, $\Phi(u) \in C\left(\left[0, t^{*}\right)\right)$, and by [6] (Cor. 7.3(ii), p. 100) the function $t \mapsto \int_{0}^{t} g(t-s)(\Phi(u))(s) \mathrm{d} s$ is 
locally absolutely continuous on $\left[0, t^{*}\right)$. Consequently, the right-hand side of $(5)$ is locally absolutely continuous on $\left[0, t^{*}\right)$ and hence, $u \in W_{\mathrm{loc}}^{1,1}\left(\left[0, t^{*}\right)\right)$.

(b) With some obvious modifications, the proof of statement (a) carries over to part (b) and is therefore omitted.

\section{Hysteresis OPERATORS}

A function $f: \mathbb{R}_{+} \rightarrow \mathbb{R}_{+}$is called a time transformation if $f$ is continuous and non-decreasing with $f(0)=0$ and $\lim _{t \rightarrow \infty} f(t)=\infty$; in other words $f$ is a time transformation if it is continuous, non-decreasing and surjective. An operator $\Phi: C\left(\mathbb{R}_{+}\right) \rightarrow C\left(\mathbb{R}_{+}\right)$is called rate independent if, for every time transformation $f$,

$$
(\Phi(u \circ f))(t)=(\Phi(u))(f(t)), \quad \forall u \in C\left(\mathbb{R}_{+}\right), \quad \forall t \in \mathbb{R}_{+} .
$$

We say that $\Phi: C\left(\mathbb{R}_{+}\right) \rightarrow C\left(\mathbb{R}_{+}\right)$is a hysteresis operator if $\Phi$ is causal and rate independent. The numerical value set $\operatorname{NVS} \Phi$ of a hysteresis operator $\Phi$ is defined by

$$
\operatorname{NVS} \Phi:=\left\{(\Phi(u))(t): u \in C\left(\mathbb{R}_{+}\right), t \in \mathbb{R}_{+}\right\} .
$$

A function $u \in C\left(\mathbb{R}_{+}\right)$is called ultimately non-decreasing if there exists $\tau \in \mathbb{R}_{+}$such that $u$ is non-decreasing on $[\tau, \infty) ; u$ is said to be approximately ultimately non-decreasing, if for all $\varepsilon>0$, there exists an ultimately non-decreasing function $v \in C\left(\mathbb{R}_{+}\right)$such that

$$
|u(t)-v(t)| \leq \varepsilon, \quad \forall t \in \mathbb{R}_{+} .
$$

In later sections, we shall invoke some or all of the following six assumptions on the hysteresis operator $\Phi$ : $C\left(\mathbb{R}_{+}\right) \rightarrow C\left(\mathbb{R}_{+}\right):$

(N1) $\Phi\left(W_{\text {loc }}^{1,1}\left(\mathbb{R}_{+}\right)\right) \subset W_{\text {loc }}^{1,1}\left(\mathbb{R}_{+}\right)$;

(N2) $\Phi$ is monotone in the sense that, for all $u \in W_{\text {loc }}^{1,1}\left(\mathbb{R}_{+}\right)$,

$$
(\Phi(u))^{\prime}(t) u^{\prime}(t) \geq 0, \quad \text { a.e. } t \in \mathbb{R}_{+} ;
$$

(N3) there exists $\lambda>0$ such that for all $\alpha \geq 0$ and $w \in C([0, \alpha])$, there exist numbers $\gamma, \delta>0$ such that

$$
\sup _{t \in[\alpha, \alpha+\gamma]}|(\Phi(u))(t)-(\Phi(v))(t)| \leq \lambda \sup _{t \in[\alpha, \alpha+\gamma]}|u(t)-v(t)|, \quad \forall u, v \in \mathcal{C}(w ; \delta, \gamma) ;
$$

(N4) for all $a>0$ and all $u \in C([0, a), \mathbb{R})$, there exist $\gamma_{1}, \gamma_{2}>0$ such that

$$
\sup _{t \in[0, \tau]}|(\Phi(u))(t)| \leq \gamma_{1}+\gamma_{2} \sup _{t \in[0, \tau]}|u(t)|, \quad \forall \tau \in[0, a) ;
$$

(N5) if $u \in C\left(\mathbb{R}_{+}\right)$is approximately ultimately non-decreasing and $\lim _{t \rightarrow \infty} u(t)=\infty$, then $\Phi(u)(t)$ and $\Phi(-u)(t)$ converge to sup NVS $\Phi$ and inf NVS $\Phi$, respectively, as $t \rightarrow \infty$;

(N6) if, for $u \in C\left(\mathbb{R}_{+}\right), \lim _{t \rightarrow \infty}(\Phi(u))(t) \in \operatorname{int} \operatorname{NVS} \Phi$, then $u$ is bounded.

Note that (N3) implies (LC). If the inequality in (N3) holds for some $\gamma>0$, then, by rate-independence, it holds for all $\gamma>0$. It is easy to see that if a hysteresis operator satisfies (N5), then NVS $\Phi$ is an interval.

An important consequence of assumptions (N1-N3) is described in the following lemma, the proof of which can be found in [9].

Lemma 3.1. If a hysteresis operator $\Phi: C\left(\mathbb{R}_{+}\right) \rightarrow C\left(\mathbb{R}_{+}\right)$satisfies $(\mathrm{N} 1-\mathrm{N} 3)$, then, for every $u \in W_{\text {loc }}^{1,1}\left(\mathbb{R}_{+}\right)$, there exists a measurable function $d_{u}: \mathbb{R}_{+} \rightarrow[0, \lambda]$ such that

$$
(\Phi(u))^{\prime}(t)=d_{u}(t) u^{\prime}(t), \quad \text { a.e. } t \in \mathbb{R}_{+} .
$$


An operator $\Phi: C\left(\mathbb{R}_{+}\right) \rightarrow C\left(\mathbb{R}_{+}\right)$is called Lipschitz continuous with Lipschitz constant $l>0$ if

$$
\sup _{t \in \mathbb{R}_{+}}|(\Phi(u))(t)-(\Phi(v))(t)| \leq l \sup _{t \in \mathbb{R}_{+}}|u(t)-v(t)|, \quad \forall u, v \in C\left(\mathbb{R}_{+}\right) .
$$

Whilst the following lemma (a proof of which can be found in [9]) will not be invoked in the paper, nevertheless it may be of independent interest as it shows, in particular, that Lipschitz continuous hysteresis operators satisfy assumptions (N1, N3) and (N4).

Lemma 3.2. Assume that $\Phi: C\left(\mathbb{R}_{+}\right) \rightarrow C\left(\mathbb{R}_{+}\right)$is a Lipschitz continuous hysteresis operator with Lipschitz constant $l>0$. Then $\Phi$ satisfies (N1, N3) (with $\lambda=l$ ) and (N4). If

$$
\lim _{t \rightarrow \infty}(\Phi(u))(t)=\sup \operatorname{NVS} \Phi \quad \text { and } \quad \lim _{t \rightarrow \infty}(\Phi(-u))(t)=\inf \operatorname{NVS} \Phi
$$

for every ultimately non-decreasing $u \in C\left(\mathbb{R}_{+}, \mathbb{R}\right)$ with $\lim _{t \rightarrow \infty} u(t)=\infty$, then $\Phi$ satisfies (N5).

In many situations, hysteresis operators occur in parametrized form, yielding a family $\left\{\Phi_{\xi}\right\}_{\xi \in P}$ of hysteresis operators, where $P$ is a subset of a normed vector space. We assume that $0 \in P$. Usually, the parameter $\xi$ plays the role of an "initial state" or is related to the initialization of the nonlinear dynamics described by $\Phi_{\xi}$ (see examples below). A family $\left\{\Phi_{\xi}\right\}_{\xi \in P}$ of hysteresis operators is called continuously unbiased at 0 (or, concisely, unbiased) if there exists a function $\psi: \mathbb{R} \times P \rightarrow \mathbb{R}_{+}$which is continuous at $(0,0)$ with $\psi(0,0)=0$ and such that

$$
\left|\left(\Phi_{\xi}(u)\right)(0)\right| \leq \psi(u(0), \xi), \quad \forall u \in C\left(\mathbb{R}_{+}\right), \forall \xi \in P .
$$

As an immediate consequence of the definition we have that, if $\left\{\Phi_{\xi}\right\}_{\xi \in P}$ is an unbiased family of hysteresis operators, then $\left(\Phi_{0}(u)\right)(0)=0$ for all $u \in C\left(\mathbb{R}_{+}\right)$with $u(0)=0$. Of course, a single hysteresis operator $\Phi$ can be interpreted as the singleton $\left\{\Phi_{0}\right\}$, where $\Phi_{0}:=\Phi$. We call $\Phi$ unbiased if $\left\{\Phi_{0}\right\}$ is unbiased.

Remark 3.3. (a) For each $\tau \in \mathbb{R}_{+}$, define the projection operator $Q_{\tau}: C\left(\mathbb{R}_{+}\right) \rightarrow C\left(\mathbb{R}_{+}\right)$by

$$
\left(Q_{\tau} u\right)(t)= \begin{cases}u(t) & \text { for } 0 \leq t \leq \tau \\ u(\tau) & \text { for } t>\tau\end{cases}
$$

An important and well-known property of hysteresis operators is that they commute with $Q_{\tau}$ for all $\tau \in \mathbb{R}_{+}$, that is, if $\Phi$ is a hysteresis operator, then

$$
\Phi Q_{\tau}=Q_{\tau} \Phi, \quad \forall \tau \in \mathbb{R}_{+} .
$$

The commutativity property (9) is an easy consequence of causality and rate-independence.

(b) We remark that there exist causal operators $\Phi: C\left(\mathbb{R}_{+}\right) \rightarrow C\left(\mathbb{R}_{+}\right)$satisfying (9), but which are not hysteresis operators. For example, consider the operator $\Phi: C\left(\mathbb{R}_{+}\right) \rightarrow C\left(\mathbb{R}_{+}\right)$defined by

$$
(\Phi(u))(t)=(1+\varphi(t)) u(t)-\int_{0}^{t} \varphi^{\prime}(s) u(s) \mathrm{d} s, \quad t \in \mathbb{R}_{+},
$$

where $\varphi: \mathbb{R}_{+} \rightarrow \mathbb{R}$ is continuously differentiable. Clearly, $\Phi$ is causal and a routine calculation shows that $\Phi$ satisfies (9). However, unless $\varphi$ is constant, $\Phi$ is not, in general, rate-independent and hence not a hysteresis operator.

(c) It is the commutativity property (9), rather than rate independence, which is used in the proofs of Lemmas 3.1 and 3.2 (see [9]). In the rest of the paper, the arguments relating to hysteresis operators are based on some or all of the assumptions (N1-N6) and on Lemma 3.1. Consequently, in the results of Sections 4 and 5, the requirement that $\Phi$ be a hysteresis operator can be weakened to the assumption that $\Phi$ is causal and satisfies (9). 
The latter assumption holds, in particular, for the operator $\Phi$ defined by $(10)$; moreover, provided that $\varphi^{\prime}(t) \geq 0$ for all $t \in \mathbb{R}_{+}, \varphi$ is bounded and $\varphi(0) \geq 0$, it is easy to show that $\Phi$ satisfies (N1-N6) ${ }^{2}$. However, we believe that non-rate-independent causal operators satisfying (9) may be of limited physical relevance and so are mainly of academic interest. For this reason, we assume that the operators under consideration are hysteresis (i.e., causal and rate independent) operators rather than causal operators satisfying (9).

In the remainder of this section, we describe various classes of hysteresis operators satisfying (N1-N6) and (LW). These classes, and the properties (N1-N3), (LC) and (LW), have a well-established pedigree (see, for example $[1,2]$ and the pioneering work in [8]).

Static nonlinearities (Nemitski operators). For a continuous function $\varphi: \mathbb{R} \rightarrow \mathbb{R}$, we define the corresponding static nonlinearity (or Nemitski operator) $\mathcal{S}^{\varphi}$ by

$$
\mathcal{S}^{\varphi}: C\left(\mathbb{R}_{+}\right) \rightarrow C\left(\mathbb{R}_{+}\right), \quad u \mapsto \varphi \circ u
$$

Clearly, $\mathcal{S}^{\varphi}$ is a hysteresis operator. The operator $\mathcal{S}^{\varphi}$ is unbiased if and only if $\varphi(0)=0$. It is easy to see that if $\varphi$ is non-decreasing and globally Lipschitz with Lipschitz constant $l \geq 0$, then $\mathcal{S}^{\varphi}$ satisfies (N1-N6) (with the constant $\lambda=l$ in (N3)). Under the additional assumption that $\varphi$ is piecewise $C^{1}$ with locally Lipschitz derivative on the intervals of continuous differentiability, a routine argument shows that $\mathcal{S}^{\varphi}$ satisfies the condition (LW). Trivially, we have that $\operatorname{NVSS}^{\varphi}=\operatorname{im} \varphi$.

Relay (passive, positive) hysteresis. Relay (also called passive or positive) hysteresis, has been discussed in a mathematically rigorous context in a number of references, see for example [9] and [11]. To give a formal definition of relay hysteresis, let $a_{0}, a_{1} \in \mathbb{R}$ with $a_{0}<a_{1}$ and let $\varphi_{0}:\left[a_{0}, \infty\right) \rightarrow \mathbb{R}$ and $\varphi_{1}:\left(-\infty, a_{1}\right] \rightarrow \mathbb{R}$ be non-decreasing and globally Lipschitz with the same Lipschitz constant $l \geq 0$ and such that $\varphi_{0}\left(a_{0}\right)=\varphi_{1}\left(a_{0}\right)$ and $\varphi_{0}\left(a_{1}\right)=\varphi_{1}\left(a_{1}\right)$. For $u \in C\left(\mathbb{R}_{+}\right)$and $t \geq 0$ define

$$
S(u, t):=u^{-1}\left(\left\{a_{0}, a_{1}\right\}\right) \cap[0, t], \quad \tau(u, t):= \begin{cases}\max S(u, t) & \text { if } S(u, t) \neq \emptyset \\ -1 & \text { if } S(u, t)=\emptyset\end{cases}
$$

Following Macki et al. [11], for $\xi \in\{0,1\}$, we define an operator $\mathcal{R}_{\xi}: C\left(\mathbb{R}_{+}\right) \rightarrow C\left(\mathbb{R}_{+}\right)$by

$$
\left(\mathcal{R}_{\xi}(u)\right)(t)= \begin{cases}\varphi_{1}(u(t)) & \text { if } u(t) \leq a_{0} \\ \varphi_{0}(u(t)) & \text { if } u(t) \geq a_{1} \\ \varphi_{1}(u(t)) & \text { if } u(t) \in\left(a_{0}, a_{1}\right), \tau(u, t) \neq-1 \text { and } u(\tau(u, t))=a_{0}, \\ \varphi_{0}(u(t)) & \text { if } u(t) \in\left(a_{0}, a_{1}\right), \tau(u, t) \neq-1 \text { and } u(\tau(u, t))=a_{1}, \\ \varphi_{0}(u(t)) & \text { if } u(t) \in\left(a_{0}, a_{1}\right), \tau(u, t)=-1 \text { and } \xi=0 \\ \varphi_{1}(u(t)) & \text { if } u(t) \in\left(a_{0}, a_{1}\right), \tau(u, t)=-1 \text { and } \xi=1\end{cases}
$$

The number $\xi$ plays the role of an "initial state" which determines the output value $\left(\mathcal{R}_{\xi}(u)\right)(t)$ if $u(s) \in\left(a_{0}, a_{1}\right)$ for all $s \in[0, t]$. The operator $\mathcal{R}_{\xi}$ is illustrated in Figure 2. It is trivial that $\mathcal{R}_{\xi}$ is a hysteresis operator. If $0 \leq a_{0}$ (respectively, $0>a_{0}$ ), then the family $\left\{\mathcal{R}_{0}, \mathcal{R}_{1}\right\}$ is unbiased if and only if $\varphi_{1}(0)=0$ (respectively, $\left.\varphi_{0}(0)=0\right)$. It is clear that $\mathcal{R}_{\xi}$ is Lipschitz continuous if and only if $\varphi_{0}(v)=\varphi_{1}(v)$ for all $v \in\left[a_{0}, a_{1}\right]$, i.e., if and only if $\mathcal{R}_{\xi}$ "degenerates" into a static nonlinearity. From [9] we know that $\mathcal{R}_{\xi}$ satisfies (N1-N6) (with $\lambda=l$ in (N3)). Under the additional assumption that $\varphi_{0}$ and $\varphi_{1}$ are piecewise $C^{1}$ with locally Lipschitz derivatives on the intervals of continuous differentiability, a routine argument shows that $\mathcal{R}_{\xi}$ satisfies the condition (LW). We note that $\operatorname{NVS} \mathcal{R}_{\xi}=\operatorname{im} \varphi_{0} \cup \operatorname{im} \varphi_{1}$.

\footnotetext{
${ }^{2}$ We are grateful to an anonymous reviewer who drew our attention to the operator $\Phi$ in (10) as an example of a non-rateindependent causal operator satisfying (N1-N6).
} 


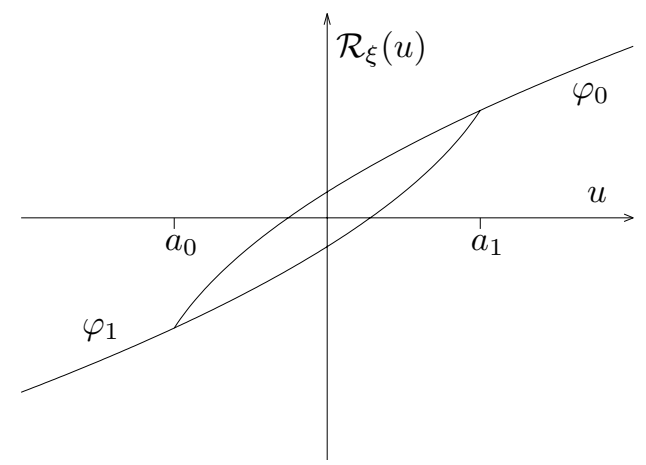

Figure 2. Relay hysteresis.

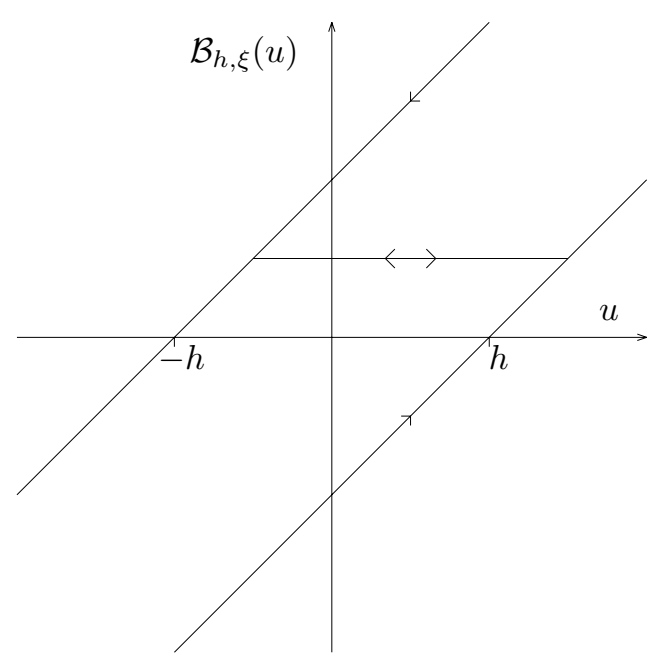

Figure 3. Backlash hysteresis.

Backlash hysteresis (play operator). A discussion of the backlash operator (also called play operator) can be found in a number of references, see for example $[1,2,8]$ and [9]. Let $h \in \mathbb{R}_{+}$and introduce the function

$$
b_{h}: \mathbb{R}^{2} \rightarrow \mathbb{R}, \quad(v, w) \mapsto \max \{v-h, \min \{v+h, w\}\}
$$

Let $C_{\mathrm{pm}}\left(\mathbb{R}_{+}\right)$denote the space of continuous piecewise monotone functions defined on $\mathbb{R}_{+}$. For all $h \in \mathbb{R}_{+}$and all $\xi \in \mathbb{R}$, we define the operator $\mathcal{B}_{h, \xi}: C_{\mathrm{pm}}\left(\mathbb{R}_{+}\right) \rightarrow C\left(\mathbb{R}_{+}\right)$by

$$
\left(\mathcal{B}_{h, \xi}(u)\right)(t)= \begin{cases}b_{h}(u(0), \xi) & \text { for } t=0 \\ b_{h}\left(u(t),\left(\mathcal{B}_{h, \xi}(u)\right)\left(t_{i}\right)\right) & \text { for } t_{i}<t \leq t_{i+1}, i=0,1,2, \ldots\end{cases}
$$

where $0=t_{0}<t_{1}<t_{2}<\ldots, \lim _{n \rightarrow \infty} t_{n}=\infty$ and $u$ is monotone on each interval $\left[t_{i}, t_{i+1}\right]$. We remark that $\xi$ plays the role of an "initial state". It is not difficult to show that the definition is independent of the choice of the partition $\left(t_{i}\right)$. Figure 3 illustrates how $\mathcal{B}_{h, \xi}$ acts. It is well-known that $\mathcal{B}_{h, \xi}$ extends to a Lipschitz continuous operator on $C\left(\mathbb{R}_{+}\right)$(with Lipschitz constant $l=1$ ), the so-called backlash operator, which we shall denote by the same symbol $\mathcal{B}_{h, \xi}$. It is also well-known (and easy to check) that $\mathcal{B}_{h, \xi}$ is a hysteresis operator. 


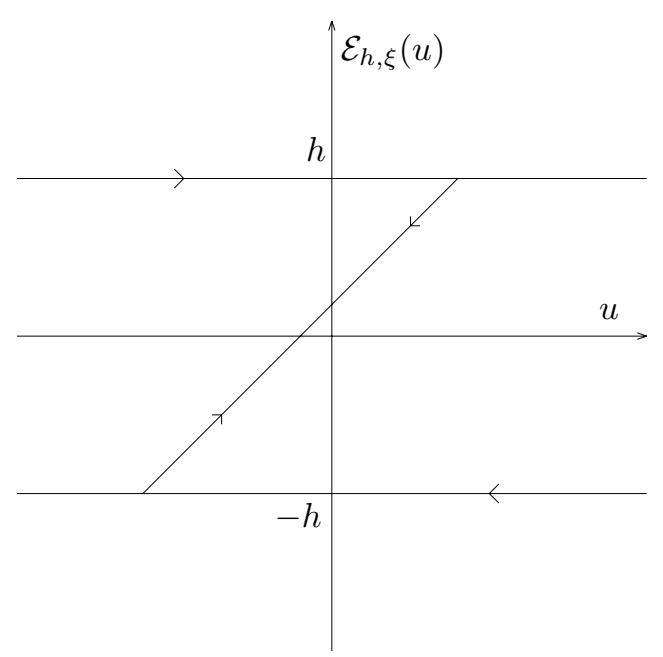

Figure 4. Elastic-plastic hysteresis.

From [2] (see Lem. 2.3.1, p. 42) we obtain

$$
\left|\mathcal{B}_{h, \xi}(u)(0)\right| \leq|u(0)|+|\xi|, \quad \forall u \in C\left(\mathbb{R}_{+}\right), \forall \xi \in \mathbb{R}
$$

showing that, for fixed $h \in \mathbb{R}_{+}$, the family $\left\{\mathcal{B}_{h, \xi}\right\}_{\xi \in \mathbb{R}}$ is unbiased. As shown in [9] for example, $\mathcal{B}_{h, \xi}$ satisfies (N1-N6) (with $\lambda=1$ in (N3)) and it follows from [2] that $\mathcal{B}_{h, \xi}$ satisfies (LW) (see Prop. 2.3.7, p. 47 in [2]). It is obvious that $\operatorname{NVS} \mathcal{B}_{h, \xi}=\mathbb{R}$.

Elastic-plastic hysteresis (stop operator). The elastic-plastic operator (also called stop operator) has been discussed in a mathematically rigorous context in a number of references, see for example $[1,2,8]$ and $[9]$. To give a formal definition of the elastic-plastic operator, for each $h \in \mathbb{R}_{+}$define the function $e_{h}: \mathbb{R} \rightarrow \mathbb{R}$ by

$$
e_{h}(v)=\min \{h, \max \{-h, v\}\}
$$

For all $h \in \mathbb{R}_{+}$and all $\xi \in \mathbb{R}$, we define an operator $\mathcal{E}_{h, \xi}: C_{\mathrm{pm}}\left(\mathbb{R}_{+}\right) \rightarrow C\left(\mathbb{R}_{+}\right)$by

$$
\left(\mathcal{E}_{h, \xi}(u)\right)(t)= \begin{cases}e_{h}(u(0)-\xi) & \text { for } t=0 \\ e_{h}\left(u(t)-u\left(t_{i}\right)+\left(\mathcal{E}_{h, \xi}(u)\right)\left(t_{i}\right)\right) & \text { for } t_{i}<t \leq t_{i+1}, i=0,1,2, \ldots\end{cases}
$$

where $0=t_{0}<t_{1}<t_{2}<\ldots, \lim _{n \rightarrow \infty} t_{n}=\infty$ and $u$ is monotone on each interval $\left[t_{i}, t_{i+1}\right]$. Again, $\xi$ plays the role of an "initial state". The operators $\mathcal{E}_{h, \xi}$ and $\mathcal{B}_{h, \xi}$ are closely related:

$$
\mathcal{E}_{h, \xi}(u)+\mathcal{B}_{h, \xi}(u)=u, \quad \forall u \in C_{\mathrm{pm}}\left(\mathbb{R}_{+}\right),
$$

see, for example [2] (p. 44). The way $\mathcal{E}_{h, \xi}$ acts is illustrated in Figure 4. It follows from (12) and the properties of $\mathcal{B}_{h, \xi}$ that $\mathcal{E}_{h, \xi}$ extends to a Lipschitz continuous hysteresis operator (with Lipschitz constant $l=2$ ). This extension, which we denote by the same symbol $\mathcal{E}_{h, \xi}$, is called the elastic plastic operator. For fixed $h \in \mathbb{R}_{+}$, the family $\left\{\mathcal{E}_{h, \xi}\right\}_{\xi \in \mathbb{R}}$ is unbiased (this follows from (12) and the fact that $\left\{\mathcal{B}_{h, \xi}\right\}_{\xi \in \mathbb{R}}$ is unbiased). As shown in [9] for example, $\mathcal{E}_{h, \xi}$ satisfies $(\mathrm{N} 1-\mathrm{N} 6)$ (with $\lambda=2$ in (N3)) and it follows from [2] that $\mathcal{E}_{h, \xi}$ satisfies (LW) (see Prop. 2.3.7, p. 47 in [2]). It is clear that $\operatorname{NVSE}_{h, \xi}=[-h, h]$.

Prandtl operators. All the hysteresis operators considered so far model relatively simple hysteresis loops. The Prandtl operator (also called the Prandtl-Ishlinskii operator), introduced below, represents a far more general 

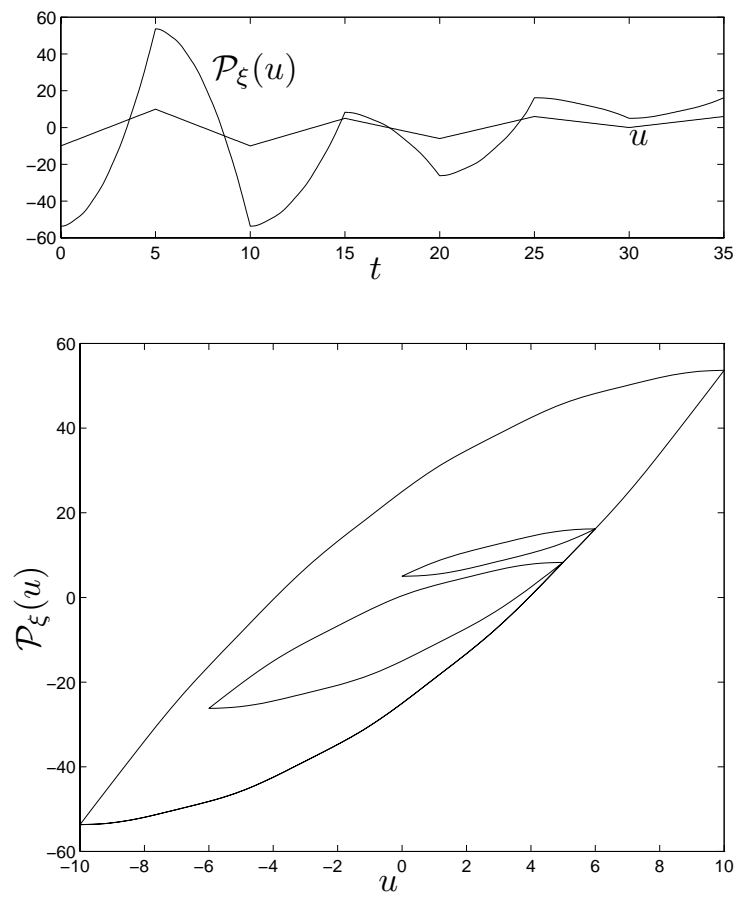

Figure 5. Example of Prandtl hysteresis.

type of hysteresis which for certain input functions exhibits nested loops in the corresponding input-output characteristics. A Preisach memory curve is a function $\xi: \mathbb{R}_{+} \rightarrow \mathbb{R}$ which has compact support and is globally Lipschitz with Lipschitz constant 1 . The set of all Preisach memory curves is denoted by $\Pi$. Furthermore, let $\mu$ be a signed Borel measure on $\mathbb{R}$ such that $|\mu|(K)<\infty$ for all compact sets $K \subset \mathbb{R}_{+}$, where $|\mu|$ denotes the total variation of $\mu$. Let $\xi \in \Pi$. The operator $\mathcal{P}_{\xi}: C\left(\mathbb{R}_{+}\right) \rightarrow C\left(\mathbb{R}_{+}\right)$defined by

$$
\left(\mathcal{P}_{\xi}(u)\right)(t)=\int_{0}^{\infty}\left(\mathcal{B}_{h, \xi(h)}(u)\right)(t) \mathrm{d} \mu(h), \quad \forall u \in C\left(\mathbb{R}_{+}, \mathbb{R}\right), \quad \forall t \in \mathbb{R}_{+}
$$

is called a Prandtl operator, $c f$. [2] (p. 54). It is well-known (and easy to check) that $\mathcal{P}_{\xi}$ is a hysteresis operator. Assume that the measure $\mu$ is finite. Then it follows from [2] that $\mathcal{P}_{\xi}$ satisfies (LW) (see Prop. 2.4.11, p. 59 in [2]). The operator $\mathcal{P}_{\xi}$ is Lipschitz continuous with Lipschitz constant $|\mu|\left(\mathbb{R}_{+}\right)$(since the backlash operator is Lipschitz continuous with Lipschitz constant 1) and, moreover, $\left\{\mathcal{P}_{\xi}\right\}_{\xi \in \Pi}$ is unbiased, since

$$
\left|\left(\mathcal{P}_{\xi}(u)\right)(0)\right| \leq|\mu|\left(\mathbb{R}_{+}\right)\left(|u(0)|+\sup _{h \geq 0}|\xi(h)|\right), \quad \forall u \in C\left(\mathbb{R}_{+}\right), \forall \xi \in \Pi
$$

by (11). Furthermore, if we additionally assume that $\mu$ is positive, then, as shown for example in [9] (N1-N6) hold (with $\lambda=\mu\left(\mathbb{R}_{+}\right)$in $\left.(\mathrm{N} 3)\right)$. For $\xi \equiv 0$ and the measure $\mu$ given by $\mu(E)=\int_{E}(\sin (\pi h)+1) \chi_{[0,10]} \mathrm{d} h$ (where $\chi_{[0,10]}$ denotes the indicator function of the interval $[0,10]$ ), the Prandtl operator is illustrated in Figure 5. It follows from [9] that $\operatorname{NVS}_{\xi}=\mathbb{R}$, provided that $\mu \neq 0$. Finally, we remark that the above class of Prandtl operators can be generalized to include the so-called Preisach operators (see [2]): a large class of such operators also satisfy (N1-N6) (see [9]) and (LW) (see [2]). 


\section{Asymptotic Behaviour of SOLUtions to FEEDBACK SYSTEMS SUBJECT TO HYSTERESIS}

Consider the feedback system shown in Figure 1, where $G$ is a convolution operator with kernel $g$, $\Phi$ is a hysteresis operator, $r_{1}$ and $r_{2}$ are input and disturbance signals, respectively. Mathematically, the feedback system is described by the integral equation

$$
y=G r_{1}+r_{2}-G \Phi(y)=g \star r_{1}+r_{2}-g \star \Phi(y) .
$$

Theorem 4.1. Let $g \in L_{\alpha}^{2}\left(\mathbb{R}_{+}\right)$for some $\alpha<0, r_{1}, r_{2} \in W_{\text {loc }}^{1,1}\left(\mathbb{R}_{+}\right)$with $r_{1}^{\prime}, r_{2}^{\prime} \in L_{\alpha}^{2}\left(\mathbb{R}_{+}\right)$, and let $\Phi$ : $C\left(\mathbb{R}_{+}\right) \rightarrow C\left(\mathbb{R}_{+}\right)$be a hysteresis operator satisfying (N1, N2) and (N3) with associated $\lambda>0$. Assume that

$$
\inf _{\omega \in \mathbb{R}} \operatorname{Re} \mathbf{G}(i \omega)>-1 / \lambda
$$

where $\mathbf{G}$ denotes the Laplace transform of $g$ (or, equivalently, $\mathbf{G}$ is the transfer function of $G$ ).

(a) If $g$ is locally of bounded variation, then (13) has a unique locally absolutely continuous solution y defined on $\mathbb{R}_{+}$(no finite escape-time) and there exist constants $\beta \in(\alpha, 0)$ and $\gamma>0$ (depending only on $g$ and $\lambda$ ) such that $y^{\prime},(\Phi(y))^{\prime} \in L_{\beta}^{2}\left(\mathbb{R}_{+}\right)$,

$$
\begin{aligned}
& \|y\|_{L^{\infty}\left(\mathbb{R}_{+}\right)}+\|\Phi(y)\|_{L^{\infty}\left(\mathbb{R}_{+}\right)}+\left\|y^{\prime}\right\|_{L_{\beta}^{2}\left(\mathbb{R}_{+}\right)}+\left\|(\Phi(y))^{\prime}\right\|_{L_{\beta}^{2}\left(\mathbb{R}_{+}\right)} \\
& \quad \leq \gamma\left(\left\|r_{1}^{\prime}\right\|_{L_{\beta}^{2}\left(\mathbb{R}_{+}\right)}+\left\|r_{2}^{\prime}\right\|_{L_{\beta}^{2}\left(\mathbb{R}_{+}\right)}+\left|r_{1}(0)\right|+\left|r_{2}(0)\right|+\left|\left(\Phi\left(r_{2}\right)\right)(0)\right|\right),
\end{aligned}
$$

and $y(t)$ and $(\Phi(y))(t)$ converge to finite limits as $t \rightarrow \infty$, the convergence being exponential with convergence rate $\beta$.

(b) If $\Phi$ satisfies (LW), then the conclusions of statement (a) remain valid.

Remark 4.2. Assume that $\left\{\Phi_{\xi}\right\}_{\xi \in P}$ is an unbiased family of hysteresis operators satisfying, for each $\xi \in P$, $(\mathrm{N} 1, \mathrm{~N} 2)$ and (N3) with associated $\lambda>0$ independent of $\xi$. Inequality (15) shows that, if

$$
\left(\left\|r_{1}^{\prime}\right\|_{L_{\beta}^{2}\left(\mathbb{R}_{+}\right)}+\left\|r_{2}^{\prime}\right\|_{L_{\beta}^{2}\left(\mathbb{R}_{+}\right)}+\left|r_{1}(0)\right|+\left|r_{2}(0)\right|+\|\xi\|\right) \rightarrow 0
$$

then

$$
\left(\|y\|_{L^{\infty}\left(\mathbb{R}_{+}\right)}+\left\|\Phi_{\xi}(y)\right\|_{L^{\infty}\left(\mathbb{R}_{+}\right)}+\left\|y^{\prime}\right\|_{L_{\beta}^{2}\left(\mathbb{R}_{+}\right)}+\left\|\left(\Phi_{\xi}(y)\right)^{\prime}\right\|_{L_{\beta}^{2}\left(\mathbb{R}_{+}\right)}\right) \rightarrow 0 .
$$

Note that this remark applies in particular to backlash, elastic-plastic and Prandtl operators.

Proof of Theorem 4.1. (a) By the positive-real assumption (14), there exists $\varepsilon>0$ such that

$$
1 / \lambda+\operatorname{Re} \mathbf{G}(i \omega) \geq \varepsilon, \quad \forall \omega \in \mathbb{R} .
$$

Setting $f(s):=\exp (-1 / \lambda-\mathbf{G}(s))$, we have

$$
|f(s)|=\exp (-1 / \lambda-\operatorname{Re} \mathbf{G}(s))
$$

Since $\mathbf{G}(s) \rightarrow 0$ as $|s| \rightarrow \infty$ in $\overline{\mathbb{C}}_{0}$ and applying the maximum modulus theorem to $f$, (16) yields

$$
1 / \lambda+\operatorname{Re} \mathbf{G}(s) \geq \varepsilon, \quad \forall s \in \overline{\mathbb{C}}_{0} .
$$


Moreover, $\mathbf{G} \in H^{\infty}\left(\mathbb{C}_{\beta}\right)$ for any $\beta>\alpha$, and therefore $\mathbf{G}$ is uniformly continuous on any vertical strip of the form $\alpha_{1} \leq \operatorname{Re} s \leq \alpha_{2}$, where $\alpha<\alpha_{1}<\alpha_{2}$ (see Th. 3.7, p. 82 in [3]). Consequently, it follows from (17) that there exists $\beta \in(\alpha, 0)$ such that

$$
1 / \lambda+\operatorname{Re} \mathbf{G}(s) \geq \varepsilon / 2>0, \quad \forall s \in \overline{\mathbb{C}}_{\beta}
$$

Now, for any $z \in \mathbb{C}$,

$$
\frac{1}{\lambda}+\operatorname{Re} z>0 \Longleftrightarrow \frac{\lambda}{2}\left|z\left(1+\frac{\lambda}{2} z\right)^{-1}\right|<1
$$

Combining this with (18) and the fact that $\mathbf{G}(s) \rightarrow 0$ as $|s| \rightarrow \infty$ in $\overline{\mathbb{C}}_{\beta}$ and setting $\nu:=\lambda / 2$, we may conclude that

$$
\kappa:=\nu \sup _{s \in \mathbb{C}_{\beta}}\left|\mathbf{G}(s)(1+\nu \mathbf{G}(s))^{-1}\right|<1
$$

The operator $\Phi$ satisfies (N3) and, hence, it also satisfies (LC). Furthermore, $g \star r_{1}+r_{2} \in W_{\text {loc }}^{1,1}\left(\mathbb{R}_{+}\right)$. Thus, it follows from part (a) of Theorem 2.2 that (13) has a unique maximal locally absolutely continuous solution $y$ defined on the maximal interval of existence $\left[0, t^{*}\right)$. Differentiation of $(13)$ shows that, on the interval $\left[0, t^{*}\right)$,

$$
y^{\prime}=\left(g \star r_{1}\right)^{\prime}+r_{2}^{\prime}-(g \star \Phi(y))^{\prime}=g \star r_{1}^{\prime}+r_{2}^{\prime}+\left[r_{1}(0)-\left(\Phi\left(r_{2}\right)\right)(0)\right] g-g \star\left(d_{y} y^{\prime}\right),
$$

where $d_{y}:\left[0, t^{*}\right) \rightarrow[0, \lambda]$ is a measurable function whose existence is guaranteed by Lemma 3.1. Defining

$$
f:=g \star r_{1}^{\prime}+r_{2}^{\prime}+\left[r_{1}(0)-\left(\Phi\left(r_{2}\right)\right)(0)\right] g \in L_{\beta}^{2}\left(\mathbb{R}_{+}\right)
$$

and setting

$$
f_{\beta}(t):=f(t) \mathrm{e}^{-\beta t}, \quad g_{\beta}(t):=g(t) \mathrm{e}^{-\beta t}, \quad y_{\beta}^{\prime}(t):=y^{\prime}(t) \mathrm{e}^{-\beta t},
$$

we obtain

$$
y_{\beta}^{\prime}=f_{\beta}-g_{\beta} \star\left(d_{y} y_{\beta}^{\prime}\right), \quad \text { on }\left[0, t^{*}\right) .
$$

This equation can be written in the form

$$
\left(\delta_{0}+\nu g_{\beta}\right) \star y_{\beta}^{\prime}=f_{\beta}-g_{\beta} \star\left[\left(d_{y}-\nu\right) y_{\beta}^{\prime}\right], \quad \text { on }\left[0, t^{*}\right),
$$

where $\delta_{0}$ denotes the unit mass at 0 . It follows from (19) that

$$
\inf _{s \in \mathbb{C}_{\beta}}|1+\nu \mathbf{G}(s)|>0,
$$

and consequently, $\delta_{0}+\nu g_{\beta}$ is invertible in the convolution algebra $\mathbb{R} \delta_{0}+L^{1}\left(\mathbb{R}_{+}\right)$(see Th. 4.1, p. 45 in $[6]$ ). Setting

$$
h:=\left(\delta_{0}+\nu g_{\beta}\right)^{-1}, \quad k:=g_{\beta} \star\left(\delta_{0}+\nu g_{\beta}\right)^{-1},
$$

we obtain from $(22)$

$$
y_{\beta}^{\prime}=h \star f_{\beta}-k \star\left[\left(d_{y}-\nu\right) y_{\beta}^{\prime}\right], \quad \text { on }\left[0, t^{*}\right) .
$$


The $L^{2}$-induced norms of the convolution operators $u \mapsto h \star u$ and $u \mapsto k \star u$ are given by

$$
\nu_{h}:=\sup _{s \in \mathbb{C}_{\beta}}\left|(1+\nu \mathbf{G}(s))^{-1}\right| \quad \text { and } \quad \nu_{k}:=\sup _{s \in \mathbb{C}_{\beta}}\left|\mathbf{G}(s)(1+\nu \mathbf{G}(s))^{-1}\right|,
$$

respectively. Since $d_{y}(t) \in[0,2 \nu]$ for a.e. $t \in\left[0, t^{*}\right)$, we have $\left|d_{y}(t)-\nu\right| \leq \nu$ for a.e. $t \in\left[0, t^{*}\right)$. Furthermore, $f_{\beta}, g_{\beta} \in L^{2}\left(\mathbb{R}_{+}\right)$and $d_{y} y_{\beta}^{\prime} \in L_{\mathrm{loc}}^{1}\left(\left[0, t^{*}\right)\right)$, and so (21) shows that $y_{\beta}^{\prime} \in L_{\mathrm{loc}}^{2}\left(\left[0, t^{*}\right)\right)$. Therefore, we may conclude from (23) that

$$
\left\|y_{\beta}^{\prime}\right\|_{L^{2}([0, t])} \leq \nu_{h}\left\|f_{\beta}\right\|_{L^{2}([0, t])}+\nu_{k} \nu\left\|y_{\beta}^{\prime}\right\|_{L^{2}([0, t])}, \quad \forall t \in\left[0, t^{*}\right) .
$$

By $(19), \nu_{k} \nu=\kappa<1$, and hence

$$
\left\|y_{\beta}^{\prime}\right\|_{L^{2}([0, t])} \leq \frac{\nu_{h}}{1-\kappa}\left\|f_{\beta}\right\|_{L^{2}([0, t])}, \quad \forall t \in\left[0, t^{*}\right) .
$$

To show that $t^{*}=\infty$, note that, by $(24), y^{\prime} \in L^{1}\left(\left[0, t^{*}\right)\right)$, and consequently $(\Phi(y))^{\prime}=d_{y} y^{\prime} \in L^{1}\left(\left[0, t^{*}\right)\right)$. It follows that $\Phi(y)$ is bounded on $\left[0, t^{*}\right)$, which implies $t^{*}=\infty$ (by part (a) of Th. 4.1). Therefore, using (24) and the fact that $\left|d_{y}(t)\right| \leq \lambda$ for a.e. $t \in \mathbb{R}_{+}$, we obtain

$$
\left\|y^{\prime}\right\|_{L_{\beta}^{2}\left(\mathbb{R}_{+}\right)}+\left\|(\Phi(y))^{\prime}\right\|_{L_{\beta}^{2}\left(\mathbb{R}_{+}\right)} \leq \frac{(1+\lambda) \nu_{h}}{1-\kappa}\|f\|_{L_{\beta}^{2}\left(\mathbb{R}_{+}\right)} .
$$

Routine estimates show that for all $t \in \mathbb{R}_{+}$

$$
\begin{aligned}
|y(t)|+|(\Phi(y))(t)| & \leq|y(0)|+|(\Phi(y))(0)|+\left\|y^{\prime}\right\|_{L^{1}\left(\mathbb{R}_{+}\right)}+\left\|(\Phi(y))^{\prime}\right\|_{L^{1}\left(\mathbb{R}_{+}\right)} \\
& \leq|y(0)|+|(\Phi(y))(0)|+\frac{1}{\sqrt{2|\beta|}}\left(\left\|y^{\prime}\right\|_{L_{\beta}^{2}\left(\mathbb{R}_{+}\right)}+\left\|(\Phi(y))^{\prime}\right\|_{L_{\beta}^{2}\left(\mathbb{R}_{+}\right)}\right) .
\end{aligned}
$$

Combining this with (25) and using that $y(0)=r_{2}(0)$ gives

$$
\|y\|_{L^{\infty}\left(\mathbb{R}_{+}\right)}+\|\Phi(y)\|_{L^{\infty}\left(\mathbb{R}_{+}\right)} \leq\left|r_{2}(0)\right|+\left|\left(\Phi\left(r_{2}\right)\right)(0)\right|+\frac{(1+\lambda) \nu_{h}}{(1-\kappa) \sqrt{2|\beta|}}\|f\|_{L_{\beta}^{2}\left(\mathbb{R}_{+}\right)},
$$

which, together with (20) and (25), yields (15). Since $y^{\prime} \in L_{\beta}^{2}\left(\mathbb{R}_{+}\right) \subset L^{1}\left(\mathbb{R}_{+}\right), y(t)$ converges to the finite limit $y^{\infty}:=y(0)+\int_{0}^{\infty} y^{\prime}(s) \mathrm{d} s$ as $t \rightarrow \infty$ and a routine estimate yields

$$
\left|y(t)-y^{\infty}\right| \leq \int_{t}^{\infty}\left|y^{\prime}(s)\right| \mathrm{d} s \leq \frac{\mathrm{e}^{\beta t}\left\|y^{\prime}\right\|_{L_{\beta}^{2}\left(\mathbb{R}_{+}\right)}}{\sqrt{2|\beta|}}, \quad \forall t \in \mathbb{R}_{+}
$$

showing that the convergence is exponential with convergence rate $\beta$. Exactly the same argument applies to show exponential convergence of $(\Phi(y))(t)$ as $t \rightarrow \infty$.

(b) The existence and uniqueness of a maximal locally absolutely continuous solution $y:\left[0, t^{*}\right) \rightarrow \mathbb{R}$ follows from part (b) of Theorem 2.2. We can argue as in the proof of part (a) to obtain inequality (24), which shows that $y^{\prime} \in L^{1}\left(\left[0, t^{*}\right)\right)$. This in turn implies that $t^{*}=\infty$, because otherwise part (b) of Theorem 2.2 would yield a contradiction to the maximality of $t^{*}$. The claims now follows as in the proof of part (a).

In Theorem 4.1 it is assumed that the linear system is described by a convolution operator with kernel in $L_{\alpha}^{2}\left(\mathbb{R}_{+}\right)$for some $\alpha<0$. This implies that the linear system is input-output stable and, in particular, does not contain any integrators. In the following we shall derive a result similar to Theorem 4.1 which applies to a class 


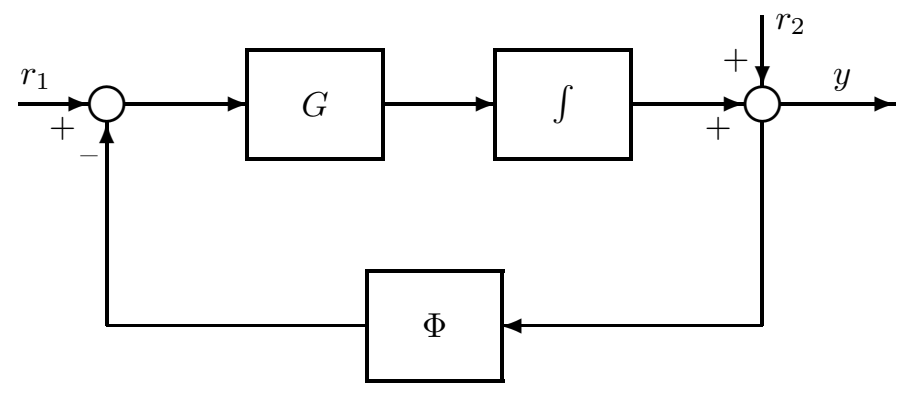

FIGURE 6. Feedback system with integrator and hysteresis.

of linear systems containing an integrator. To this end consider the feedback system shown in Figure 6 , where $G$ : $L^{2}\left(\mathbb{R}_{+}\right) \rightarrow L^{2}\left(\mathbb{R}_{+}\right)$is a linear bounded shift-invariant operator. Since shift-invariance implies causality, $G$ can be extended to a shift-invariant operator mapping $L_{\text {loc }}^{2}\left(\mathbb{R}_{+}\right)$into itself. We will not distinguish notationally between $G$ and its extension. We define a shift-invariant operator $H: L_{\text {loc }}^{2}\left(\mathbb{R}_{+}\right) \rightarrow L_{\text {loc }}^{2}\left(\mathbb{R}_{+}\right)$by

$$
H u=\int_{0}^{\cdot} G u .
$$

Denoting the transfer functions of $G$ and $H$ by $\mathbf{G}$ and $\mathbf{H}$, respectively, we have that $\mathbf{G} \in H^{\infty}\left(\mathbb{C}_{0}\right)$ and $\mathbf{H}(s)=\mathbf{G}(s) / s$. We assume that

(L) the limit $\mathbf{G}(0):=\lim _{s \rightarrow 0, s \in \mathbb{C}_{0}} \mathbf{G}(s)$ exists, $\mathbf{G}(0)>0$ and

$$
\limsup _{s \rightarrow 0, s \in \mathbb{C}_{0}}|(\mathbf{G}(s)-\mathbf{G}(0)) / s|<\infty .
$$

The proof of the following lemma can be found in the Appendix.

Lemma 4.3. Let $G \in \mathcal{L}\left(L^{2}\left(\mathbb{R}_{+}\right)\right)$be a shift-invariant operator whose transfer function $\mathbf{G}$ satisfies condition (L). Then

(a) $G \theta-\mathbf{G}(0) \in L^{2}\left(\mathbb{R}_{+}\right)$, where $\theta$ denotes the unit-step function;

(b) the shift-invariant operator $K$ defined on $L^{2}\left(\mathbb{R}_{+}\right)$by

$$
(K u)(t)=\int_{0}^{t}((G u)(s)-\mathbf{G}(0) u(s)) \mathrm{d} s
$$

is in $\mathcal{L}\left(L^{2}\left(\mathbb{R}_{+}\right)\right)$with convolution kernel in $L^{2}\left(\mathbb{R}_{+}\right)$;

(c) for $u \in W_{\text {loc }}^{1,1}\left(\mathbb{R}_{+}\right)$with $u^{\prime} \in L^{2}\left(\mathbb{R}_{+}\right)$, we have that

$$
G u=\mathbf{G}(0) u+K u^{\prime}+u(0)(G \theta-\mathbf{G}(0)) ;
$$

in particular, $G u-\mathbf{G}(0) u \in L^{2}\left(\mathbb{R}_{+}\right)$.

Consider the integral equation

$$
y=H r_{1}+r_{2}-H \Phi(y)
$$

which describes the feedback system shown in Figure 6 . 
Lemma 4.4. Assume that $H$ is of the form (26), where $G: L^{2}\left(\mathbb{R}_{+}\right) \rightarrow L^{2}\left(\mathbb{R}_{+}\right)$is a linear bounded shiftinvariant operator. Let $\Phi: C\left(\mathbb{R}_{+}\right) \rightarrow C\left(\mathbb{R}_{+}\right)$be a hysteresis operator satisfying (LC) and (N4). Moreover, let $r_{1} \in L_{\mathrm{loc}}^{2}\left(\mathbb{R}_{+}\right)$and $r_{2} \in W_{\mathrm{loc}}^{1,2}\left(\mathbb{R}_{+}\right)$. Then $(29)$ has a unique locally absolutely continuous solution $y$ defined on $\mathbb{R}_{+}$(no finite escape-time).

Proof. Let $\beta>0$. Since the transfer function $\mathbf{H}$ is of the form $\mathbf{H}(s)=\mathbf{G}(s) / s$, where $\mathbf{G} \in H^{\infty}\left(\mathbb{C}_{0}\right)$, it is clear that $\mathbf{H} \in H^{2}\left(\mathbb{C}_{\beta}\right)$. Consequently, by a well-known theorem of Paley and Wiener, the inverse Laplace transform of $\mathbf{H}$, denoted by $h$, is in $L_{\beta}^{2}\left(\mathbb{R}_{+}\right) \subset L_{\text {loc }}^{1}\left(\mathbb{R}_{+}\right)$. Setting $r:=H r_{1}+r_{2} \in W_{\text {loc }}^{1,2}\left(\mathbb{R}_{+}\right),(29)$ can be rewritten in the form

$$
y(t)=r(t)-\int_{0}^{t} h(t-s)(\Phi(y))(s) \mathrm{d} s, \quad t \geq 0 .
$$

Thus it follows from part (a) of Theorem 2.2 that (29) has a unique maximal solution $y \in C\left(\left[0, t^{*}\right)\right)$. It is clear that $r$ and $H \Phi(y)$ are locally absolutely continuous and hence so is $y=r-H \Phi(y)$. To show that $t^{*}=\infty$, suppose, for contradiction, that $t^{*}<\infty$. Using assumption (N4) and arguing as in [9] (see Step 3 in the proof of Lem. 14.5, p. 290 in [9]), shows that $y$ is bounded on $\left[0, t^{*}\right)$. Invoking again (N4), we may conclude that $\Phi(y)$ is bounded on $\left[0, t^{*}\right)$, which, by part (a) of Theorem 2.2 , contradicts the supposition that $t^{*}$ is finite.

Theorem 4.5. Assume that $H$ is of the form $(26)$, where $G: L^{2}\left(\mathbb{R}_{+}\right) \rightarrow L^{2}\left(\mathbb{R}_{+}\right)$is a linear bounded shiftinvariant operator with transfer function $\mathbf{G}$ satisfying assumption $(\mathrm{L})$. Let $\Phi: C\left(\mathbb{R}_{+}\right) \rightarrow C\left(\mathbb{R}_{+}\right)$be a hysteresis operator satisfying (N1-N5) and such that $0 \in \operatorname{clos}$ NVS $\Phi$. Let $\lambda>0$ be the constant associated with (N3). Assume that there exists $\varepsilon>0$ such that

$$
\operatorname{Re} \mathbf{H}(i \omega)=\operatorname{Re}(\mathbf{G}(i \omega) / i \omega) \geq \varepsilon-1 / \lambda, \quad \text { a.e. } \omega \in \mathbb{R}
$$

Then, for all $r_{1} \in L^{2}\left(\mathbb{R}_{+}\right)$and all $r_{2} \in W_{\text {loc }}^{1,1}\left(\mathbb{R}_{+}\right)$with $r_{2}^{\prime} \in L^{2}\left(\mathbb{R}_{+}\right)$, there exists a unique locally absolutely continuous solution $y$ of $(29)$ defined on $\mathbb{R}_{+}$and such that $(\Phi(y))^{\prime} \in L^{2}\left(\mathbb{R}_{+}\right)$, $\Phi(y)$ is bounded and $\lim _{t \rightarrow \infty}(\Phi(y))(t)=0$. Moreover, there exists $\gamma>0$ (depending only on $G, \lambda$ and $\varepsilon$ ) such that

$$
\|\Phi(y)\|_{L^{\infty}\left(\mathbb{R}_{+}\right)}+\left\|(\Phi(y))^{\prime}\right\|_{L^{2}\left(\mathbb{R}_{+}\right)} \leq \gamma\left(\left\|r_{1}\right\|_{L^{2}\left(\mathbb{R}_{+}\right)}+\left\|r_{2}^{\prime}\right\|_{L^{2}\left(\mathbb{R}_{+}\right)}+\left|\left(\Phi\left(r_{2}\right)\right)(0)\right|\right) .
$$

Under the additional assumptions that (N6) holds and that $0 \in \operatorname{int} \operatorname{NVS} \Phi, y$ is bounded.

For the proof of Theorem 4.5 we need the following lemma which is a special case of Theorem 3.3 in [4].

Lemma 4.6. Assume that $H$ is of the form (26), where $G: L^{2}\left(\mathbb{R}_{+}\right) \rightarrow L^{2}\left(\mathbb{R}_{+}\right)$is a linear bounded shiftinvariant operator with transfer function $\mathbf{G}$ satisfying assumption $(\mathrm{L})$. Consider the integral equation

$$
w=r-H \varphi(\cdot, w)
$$

where $r \in L^{2}\left(\mathbb{R}_{+}\right)+\mathbb{R}$ and $\varphi: \mathbb{R}_{+} \times \mathbb{R} \rightarrow \mathbb{R}$ is a time-dependent static nonlinearity satisfying the sector condition

$$
0 \leq \varphi(t, v) v \leq a v^{2}, \quad \forall v \in \mathbb{R}, \text { a.e. } t \in \mathbb{R}_{+}
$$

for some $a>0$. Assume that there exists $\varepsilon>0$ such that

$$
\operatorname{Re} \mathbf{H}(i \omega)=\operatorname{Re}(\mathbf{G}(i \omega) / i \omega) \geq \varepsilon-1 / a, \quad \text { a.e. } \omega \in \mathbb{R}
$$

where $\mathbf{H}$ and $\mathbf{G}$ denote the transfer functions of $H$ and $G$, respectively. If $w: \mathbb{R}_{+} \rightarrow \mathbb{R}$ is a solution of (32), then (i) $\varphi(\cdot, w) \in L^{2}\left(\mathbb{R}_{+}\right)$, (ii) $\lim _{t \rightarrow \infty} \int_{0}^{t} \varphi(s, w(s)) \mathrm{d}$ s exists and is finite, and (iii) there exists $\gamma>0$ (depending 
only on $G$, a and $\varepsilon$ ) such that

$$
\|\varphi(\cdot, w)\|_{L^{2}\left(\mathbb{R}_{+}\right)}+\sup _{t \in \mathbb{R}_{+}}\left|\int_{0}^{t} \varphi(s, w(s)) \mathrm{d} s\right| \leq \gamma\left(\left\|r-r_{0}\right\|_{L^{2}\left(\mathbb{R}_{+}\right)}+\left|r_{0}\right|\right)
$$

where $r_{0} \in \mathbb{R}$ is such that $r-r_{0} \in L^{2}\left(\mathbb{R}_{+}\right)$.

Proof of Theorem 4.5. Since (N3) implies (LC), we may apply Lemma 4.4 to obtain the existence of a unique locally absolutely continuous solution $y$ of $(32)$ defined on $\mathbb{R}_{+}$. Setting $f:=H r_{1}+r_{2} \in W_{\text {loc }}^{1,2}\left(\mathbb{R}_{+}\right)$, we have $y=f-H \Phi(y)$ and hence

$$
y^{\prime}=f^{\prime}-G \Phi(y)
$$

By Lemma 3.1 there exists a measurable function $d_{y}: \mathbb{R}_{+} \rightarrow[0, \lambda]$ such that

$$
(\Phi(y))^{\prime}=d_{y} y^{\prime}=-d_{y} w
$$

where

$$
w:=-y^{\prime}
$$

Integrating (35) from 0 to $t$ leads to

$$
(\Phi(y))(t)=(\Phi(y))(0)-\int_{0}^{t} d_{y}(s) w(s) \mathrm{d} s
$$

Define

$$
r:=G((\Phi(y))(0) \theta)-f^{\prime}=G((\Phi(y))(0) \theta)-G r_{1}-r_{2}^{\prime}
$$

where $\theta: \mathbb{R} \rightarrow \mathbb{R}$ denotes the unit-step function. Combining (34,36) and (37) and using the fact that, by shift-invariance, $G$ commutes with integration, we obtain

$$
w(t)=r(t)-\int_{0}^{t}\left(G\left(d_{y} w\right)\right)(s) \mathrm{d} s=r(t)-\left(H\left(d_{y} w\right)\right)(t) .
$$

Note that (38) is of the form (32) with $\varphi$ given by $\varphi(t, v)=d_{y}(t) v$ satisfying the sector condition $0 \leq \varphi(t, v) v$ $\leq \lambda v^{2}$ for all $v \in \mathbb{R}$ and almost all $t \in \mathbb{R}_{+}$. Moreover, using the facts that $r_{1}, r_{2}^{\prime} \in L^{2}\left(\mathbb{R}_{+}\right)$, that $G: L^{2}\left(\mathbb{R}_{+}\right)$ $\rightarrow L^{2}\left(\mathbb{R}_{+}\right)$is bounded and that, by part (a) of Lemma $4.3, G \theta-\mathbf{G}(0) \in L^{2}\left(\mathbb{R}_{+}\right)$, we conclude that $r \in L^{2}\left(\mathbb{R}_{+}\right)+$ $\mathbb{R}$. We may now apply Lemma 4.6 to $(38)$ to conclude that $d_{y} w \in L^{2}\left(\mathbb{R}_{+}\right)$and that $\int_{0}^{t} d_{y}(s) w(s) \mathrm{d} s$ converges to a finite limit as $t \rightarrow \infty$. Using (35) and (36) it follows that $(\Phi(y))^{\prime} \in L^{2}\left(\mathbb{R}_{+}\right)$and that $\lim _{t \rightarrow \infty}(\Phi(y))(t)$ exists and is finite. It follows from (37) and part (a) of Lemma 4.3 that

$$
r-\left(\Phi\left(r_{2}\right)\right)(0) \mathbf{G}(0)=\left(\Phi\left(r_{2}\right)\right)(0)(G \theta-\mathbf{G}(0))-G r_{1}-r_{2}^{\prime} \in L^{2}\left(\mathbb{R}_{+}\right),
$$

and therefore (31) is an immediate consequence of $(33)$.

Setting $l:=\lim _{t \rightarrow \infty}(\Phi(y))(t)$, it remains to show that $l=0$. To this end write $y^{\prime}=f^{\prime}-G \Phi(y)$ in the form

$$
y^{\prime}=w_{1}+w_{2}, \quad \text { where } \quad w_{1}:=-\mathbf{G}(0) \Phi(y), w_{2}:=f^{\prime}-(G \Phi(y)-\mathbf{G}(0) \Phi(y)) .
$$


Clearly,

$$
\lim _{t \rightarrow \infty} w_{1}(t)=-\mathbf{G}(0) l .
$$

Since $\Phi(y) \in W_{\text {loc }}^{1,1}\left(\mathbb{R}_{+}\right)$with $(\Phi(y))^{\prime} \in L^{2}\left(\mathbb{R}_{+}\right)$, it follows from part (c) of Lemma 4.3 that $G \Phi(y)-\mathbf{G}(0) \Phi(y)$ $\in L^{2}\left(\mathbb{R}_{+}\right)$. Moreover, since also $f^{\prime} \in L^{2}\left(\mathbb{R}_{+}\right)$, we have that $w_{2} \in L^{2}\left(\mathbb{R}_{+}\right)$. Seeking a contradiction, suppose that $l<0$. By assumption $(\mathrm{L}), \mathbf{G}(0)>0$, and so $\delta:=-\mathbf{G}(0) l>0$. We use the splitting $y^{\prime}=w_{1}+w_{2}$ and we further split $w_{2}$ into $w_{2}=w_{3}+w_{4}$, where

$$
w_{3}(t):=\max \left\{w_{2}(t),-\delta / 3\right\}, \quad w_{4}(t):=w_{2}(t)-w_{3}(t), \quad \forall t \in \mathbb{R}_{+} .
$$

Thus, $w_{3} \geq-\delta / 3$ and $w_{4}=\chi_{E}\left(w_{2}+\delta / 3\right)$, where $\chi_{E}$ is the indicator function of the set $E:=\left\{t \in \mathbb{R}_{+} \mid w_{2}(t)\right.$ $<-\delta / 3\}$. In particular, since $w_{2} \in L^{2}\left(\mathbb{R}_{+}\right)$, the set $E$ has finite Lebesgue measure, and so, $w_{4} \in L^{1}\left(\mathbb{R}_{+}\right)$. Since $w_{3} \geq-\delta / 3$ and $\lim _{t \rightarrow \infty} w_{1}(t)=\delta$, by taking $\tau_{0} \geq 0$ large enough, we have

$$
w_{1}(t)+w_{3}(t) \geq \delta / 3, \quad \forall t \geq \tau_{0} .
$$

Integrating the equation $y^{\prime}=w_{1}+w_{3}+w_{4}$ from $\tau \geq \tau_{0}$ to $t$ gives

$$
y(t)=y(\tau)+\int_{\tau}^{t}\left(w_{1}(s)+w_{3}(s)\right) \mathrm{d} s+\int_{\tau}^{t} w_{4}(s) \mathrm{d} s \geq y(\tau)+\delta(t-\tau) / 3+\int_{\tau}^{t} w_{4}(s) \mathrm{d} s .
$$

Since $w_{4} \in L^{1}\left(\mathbb{R}_{+}\right)$, for given $\varepsilon>0$, there exists $\tau_{\varepsilon} \geq \tau_{0}$ such that

$$
\int_{\tau_{\varepsilon}}^{\infty}\left|w_{4}(s)\right| \mathrm{d} s \leq \varepsilon
$$

Defining $y_{\varepsilon} \in C\left(\mathbb{R}_{+}\right)$by

$$
y_{\varepsilon}(t)= \begin{cases}y(t) & \text { for } 0 \leq t \leq \tau_{\varepsilon} \\ y\left(\tau_{\varepsilon}\right)+\int_{\tau_{\varepsilon}}^{t}\left(w_{1}(s)+w_{3}(s)\right) \mathrm{d} s & \text { for } t>\tau_{\varepsilon}\end{cases}
$$

it follows from (39) that $y_{\varepsilon}$ is ultimately non-decreasing, and moreover, by (40) and (41),

$$
\left|y(t)-y_{\varepsilon}(t)\right| \leq \varepsilon, \quad \forall t \in \mathbb{R}_{+},
$$

showing that $y$ is approximately ultimately non-decreasing. By $(40), \lim _{t \rightarrow \infty} y(t)=\infty$, and so we may invoke (N5) to conclude that

$$
0>l=\lim _{t \rightarrow \infty}(\Phi(y))(t)=\sup \operatorname{NVS} \Phi,
$$

which is in contradiction to $0 \in \operatorname{clos} \operatorname{NVS} \Phi$. Therefore, $l \geq 0$. An analogous contradiction argument shows that $l \leq 0$. Therefore, $l=0$ and so $\lim _{t \rightarrow \infty}(\Phi(y))(t)=0$. Boundedness of $y$ now follows immediately, provided (N6) holds and $0 \in \operatorname{int} \operatorname{NVS} \Phi$.

\section{Application to Well-Posed state-space systems}

This section is devoted to applications of the results in Section 4 to well-posed state-space systems. There are a number of equivalent definitions of well-posed systems, see [5, 13-18,20,21]. We will be brief in the following and refer the reader to the above references for more details. Throughout this section, we shall be considering 
a well-posed system $\Sigma$ with state-space $X$, input space $\mathbb{R}$ and output space $\mathbb{R}$, generating operators $(A, B, C)$, input-output operator $G$ and transfer function $\mathbf{G}$. Here $X$ is a real Hilbert space with norm denoted by $\|\cdot\|$, $A$ is the generator of a strongly continuous semigroup $\mathbf{T}=\left(\mathbf{T}_{t}\right)_{t \geq 0}$ on $X, B \in \mathcal{L}\left(\mathbb{R}, X_{-1}\right)$ and $C \in \mathcal{L}\left(X_{1}, \mathbb{R}\right)$, where $X_{1}$ denotes the space $\operatorname{dom}(A)$ endowed with the norm $\|x\|_{1}:=\|x\|+\|A x\|$ (the graph norm of $A$ ), whilst $X_{-1}$ denotes the completion of $X$ with respect to the norm $\|x\|_{-1}=\left\|(z I-A)^{-1} x\right\|$, where $z \in \varrho(A)$ (different choices of $z$ lead to equivalent norms). Clearly, $X_{1} \subset X \subset X_{-1}$ and the canonical injections are bounded and dense. The semigroup $\mathbf{T}$ restricts to a strongly continuous semigroup on $X_{1}$ and extends to a strongly continuous semigroup on $X_{-1}$ with the exponential growth constant being the same on all three spaces; the generator of the restriction (extension) of $\mathbf{T}$ is a restriction (extension) of $A$; we shall use the same symbol $\mathbf{T}$ (respectively, $A$ ) for the original semigroup (respectively, generator) and the associated restrictions and extensions: with this convention, we may write $A \in \mathcal{L}\left(X, X_{-1}\right)$ (considered as a generator on $X_{-1}$, the domain of $A$ is $X$ ). Moreover, the operator $B$ is an admissible control operator for $\mathbf{T}$, i.e., for each $t \in \mathbb{R}_{+}$there exists $\alpha_{t} \geq 0$ such that

$$
\left\|\int_{0}^{t} \mathbf{T}_{t-\tau} B u(\tau) \mathrm{d} \tau\right\| \leq \alpha_{t}\|u\|_{L^{2}([0, t])}, \quad \forall u \in L^{2}([0, t]) .
$$

The operator $C$ is an admissible observation operator for $\mathbf{T}$, i.e., for each $t \in \mathbb{R}_{+}$there exists $\beta_{t} \geq 0$ such that

$$
\left(\int_{0}^{t}\left\|C \mathbf{T}_{\tau} x\right\|^{2} \mathrm{~d} \tau\right)^{1 / 2} \leq \beta_{t}\|x\|, \quad \forall x \in X_{1}
$$

The control operator $B$ is said to be bounded if it is so as a map from the input space $\mathbb{R}$ to the state space $X$, otherwise is said to be unbounded; the observation operator $C$ is said to be bounded if it can be extended continuously to $X$, otherwise, $C$ is said to be unbounded.

The so-called $\Lambda$-extension $C_{\Lambda}$ of $C$ is defined by

$$
C_{\Lambda} x=\lim _{s \rightarrow \infty, s \in \mathbb{R}} C s(s I-A)^{-1} x
$$

with $\operatorname{dom}\left(C_{\Lambda}\right)$ consisting of all $x \in X$ for which the above limit exists. For every $x \in X, \mathbf{T}_{t} x \in \operatorname{dom}\left(C_{\Lambda}\right)$ for a.e. $t \in \mathbb{R}_{+}$and, if $\omega>\omega(\mathbf{T})$, then $C_{\Lambda} \mathbf{T} x \in L_{\omega}^{2}\left(\mathbb{R}_{+}\right)$, where

$$
\omega(\mathbf{T}):=\lim _{t \rightarrow \infty} \frac{1}{t} \ln \left\|\mathbf{T}_{t}\right\|
$$

denotes the exponential growth constant of $\mathbf{T}$. The transfer function $\mathbf{G}$ satisfies

$$
\frac{1}{s-z}(\mathbf{G}(s)-\mathbf{G}(z))=-C(s I-A)^{-1}(z I-A)^{-1} B, \quad \forall s, z \in \mathbb{C}_{\omega(\mathbf{T})}, s \neq z,
$$

and $\mathbf{G} \in H^{\infty}\left(\mathbb{C}_{\omega}\right)$ for every $\omega>\omega(\mathbf{T})$. The input-output operator $G: L_{\text {loc }}^{2}\left(\mathbb{R}_{+}\right) \rightarrow L_{\text {loc }}^{2}\left(\mathbb{R}_{+}\right)$is continuous and shift-invariant; moreover, for every $\omega>\omega(\mathbf{T}), G \in \mathcal{L}\left(L_{\omega}^{2}\left(\mathbb{R}_{+}\right)\right)$and

$$
(\widehat{G u})(s)=\mathbf{G}(s) \hat{u}(s), \quad \forall s \in \mathbb{C}_{\omega}, \forall u \in L_{\omega}^{2}\left(\mathbb{R}_{+}\right),
$$

where the superscript ^ denotes the Laplace transform. In the following, let $z \in \mathbb{C}_{\omega(\mathbf{T})}$ be fixed, but arbitrary. For $x^{0} \in X$ and $u \in L_{\text {loc }}^{2}\left(\mathbb{R}_{+}\right)$, let $x$ and $y$ denote the state and output functions of $\Sigma$, respectively, corresponding to the initial condition $x(0)=x^{0} \in X$ and the input function $u$. Then $x(t)=\mathbf{T}_{t} x^{0}+\int_{0}^{t} \mathbf{T}_{t-\tau} B u(\tau) \mathrm{d} \tau$ for all $t \in \mathbb{R}_{+}, x(t)-(z I-A)^{-1} B u(t) \in \operatorname{dom}\left(C_{\Lambda}\right)$ for a.e. $t \in \mathbb{R}_{+}$and

$$
\begin{aligned}
x^{\prime}(t) & =A x(t)+B u(t), \quad x(0)=x^{0}, \quad \text { a.e. } t \in \mathbb{R}_{+}, \\
y(t) & =C_{\Lambda}\left(x(t)-(z I-A)^{-1} B u(t)\right)+\mathbf{G}(z) u(t), \quad \text { a.e. } t \geq 0 .
\end{aligned}
$$


Of course, the differential equation (43a) has to be interpreted in $X_{-1}$. Note that the output equation (43b) yields the following formula for the input-output operator $G$

$$
\begin{aligned}
(G u)(t)=C_{\Lambda}\left[\int_{0}^{t} \mathbf{T}_{t-\tau} B u(\tau) \mathrm{d} \tau-(z I-A)^{-1} B u(t)\right] & +\mathbf{G}(z) u(t), \\
\forall u & \in L_{\text {loc }}^{2}\left(\mathbb{R}_{+}\right), \text {a.e. } t \in \mathbb{R}_{+} .
\end{aligned}
$$

In the following, we identify $\Sigma$ and (43) and refer to (43) as a well-posed system.

We say that (43) is exponentially stable if $\omega(\mathbf{T})<0$. The well-posed system (43) is called strongly stable if the following four conditions are satisfied:

(i) $G$ is $L^{2}$-stable, i.e., $G \in \mathcal{L}\left(L^{2}\left(\mathbb{R}_{+}\right)\right)$, or, equivalently, $\mathbf{G} \in H^{\infty}\left(\mathbb{C}_{0}\right)$;

(ii) $\mathbf{T}$ is strongly stable, i.e., $\lim _{t \rightarrow \infty} \mathbf{T}_{t} x=0$ for all $x \in X$;

(iii) $B$ is an infinite-time admissible control operator, i.e., there exists $\alpha \geq 0$ such that $\left\|\int_{0}^{\infty} \mathbf{T}_{s} B v(s) \mathrm{d} s\right\|$ $\leq \alpha\|v\|_{L^{2}\left(\mathbb{R}_{+}\right)}$for all $v \in L^{2}\left(\mathbb{R}_{+}\right)$

(iv) $C$ is an infinite-time admissible observation operator, i.e., there exists $\beta \geq 0$ such that $\left(\int_{0}^{\infty}\left\|C \mathbf{T}_{s} x\right\|^{2} \mathrm{~d} s\right)^{1 / 2}$ $\leq \beta\|x\|$ for all $x \in X_{1}$.

Obviously, exponential stability implies strong stability, but the converse is not true.

If the well-posed system (43) is regular, i.e., the following limit

$$
\lim _{s \rightarrow \infty, s \in \mathbb{R}} \mathbf{G}(s)=D
$$

exists and is finite, then $x(t) \in \operatorname{dom}\left(C_{\Lambda}\right)$ for a.e. $t \in \mathbb{R}_{+}$, the output equation (43b) simplifies to

$$
y(t)=C_{\Lambda} x(t)+D u(t), \quad \text { a.e. } t \geq 0
$$

and

$$
(G u)(t)=C_{\Lambda} \int_{0}^{t} \mathbf{T}_{t-\tau} B u(\tau) \mathrm{d} \tau+D u(t), \quad \forall u \in L_{\text {loc }}^{2}\left(\mathbb{R}_{+}\right), \text {a.e. } t \in \mathbb{R}_{+} .
$$

Moreover, in the regular case, we have that $(s I-A)^{-1} B \mathbb{R} \subset \operatorname{dom}\left(C_{\Lambda}\right)$ for all $s \in \varrho(A)$ and

$$
\mathbf{G}(s)=C_{\Lambda}(s I-A)^{-1} B+D, \quad \forall s \in \mathbb{C}_{\omega(\mathbf{T})} .
$$

The number $D$ is called the feedthrough of (43).

Finally, for the application of the results in Section 4 to well-posed systems, we need the following two technical lemmas:

Lemma 5.1. Assume that the control operator $B$ or the observation operator $C$ is bounded. Then system (43) is regular. Moreover, the inverse Laplace transform of the transfer function $\mathbf{G}$, or, equivalently, the impulse response of (43), is in $\mathbb{R} \delta_{0}+L_{\omega}^{2}\left(\mathbb{R}_{+}\right)$for any $\omega>\omega(\mathbf{T})$, where $\delta_{0}$ denotes the unit mass at 0 .

The proof of the above lemma can be found in Logemann and Ryan [10], whilst the proof of the lemma below is given in the Appendix.

Lemma 5.2. (a) Assume that $\mathbf{T}$ is exponentially stable, i.e. $\omega(\mathbf{T})<0$ and let $\beta \in(\omega(\mathbf{T}), 0)$. Then there exists $\gamma>0$ such that, for all $x^{0} \in X$ and all $u \in W_{\text {loc }}^{1,1}\left(\mathbb{R}_{+}\right)$with $u^{\prime} \in L_{\beta}^{2}\left(\mathbb{R}_{+}\right)$, the solution $x$ of (43a) satisfies

$$
\left\|x+A^{-1} B u\right\|_{L_{\beta}^{\infty}\left(\mathbb{R}_{+}, X\right)} \leq \gamma\left(\left\|x^{0}\right\|+|u(0)|+\left\|u^{\prime}\right\|_{L_{\beta}^{2}\left(\mathbb{R}_{+}\right)}\right) .
$$


(b) Assume that $\mathbf{T}$ is strongly stable, $0 \in \varrho(A)$ and $B$ is infinite-time admissible. Then there exists $\gamma>0$ such that, for all $x^{0} \in X$ and all $u \in W_{\mathrm{loc}}^{1,1}\left(\mathbb{R}_{+}\right)$with $u^{\prime} \in L^{2}\left(\mathbb{R}_{+}\right)$, the solution $x$ of (43a) satisfies

$$
\left\|x+A^{-1} B u\right\|_{L^{\infty}\left(\mathbb{R}_{+}, X\right)} \leq \gamma\left(\left\|x^{0}\right\|+|u(0)|+\left\|u^{\prime}\right\|_{L^{2}\left(\mathbb{R}_{+}\right)}\right)
$$

Moreover, $\lim _{t \rightarrow \infty}\left\|x(t)+A^{-1} B u(t)\right\|=0$.

Application of Theorem 4.1. Assume that (43) is regular with feedthrough $D=0$ and consider the feedback law $u=-\Phi(y)$, where $\Phi: C\left(\mathbb{R}_{+}\right) \rightarrow C\left(\mathbb{R}_{+}\right)$is a hysteresis operator. The feedback system is then given by

$$
x^{\prime}=A x-B \Phi\left(C_{\Lambda} x\right), \quad x(0)=x^{0} \in X .
$$

Let $0<\tau \leq \infty$. A solution of $(45)$ on the interval $[0, \tau)$ is a continuous function $x:[0, \tau) \rightarrow X$ such that $x(t) \in$ $\operatorname{dom}\left(C_{\Lambda}\right)$ for a.e. $t \in[0, \tau), C_{\Lambda} x \in C([0, \tau))$ and

$$
x(t)=x^{0}+\int_{0}^{t}\left[A x(s)-B\left(\Phi\left(C_{\Lambda} x\right)\right)(s)\right] \mathrm{d} s, \quad \forall t \in[0, \tau) .
$$

Theorem 5.3. Assume that (43) is exponentially stable, that $B$ or $C$ is bounded (hence (43) is regular, by Lem. 5.1) and that the feedthrough is zero. Furthermore assume that $\Phi: C\left(\mathbb{R}_{+}\right) \rightarrow C\left(\mathbb{R}_{+}\right)$is a hysteresis operator satisfying $(\mathrm{LW})$ and $(\mathrm{N} 1, \mathrm{~N} 2)$ and $(\mathrm{N} 3)$ with associated $\lambda>0$. Assume that

$$
\inf _{\omega \in \mathbb{R}} \operatorname{Re} \mathbf{G}(i \omega)>-1 / \lambda
$$

Then, for all $x^{0} \in X_{1}$, equation (45) has a unique solution $x$ defined on $\mathbb{R}_{+}$satisfying $x(t) \in \operatorname{dom}(C)$ for all $t \in \mathbb{R}_{+}$. Moreover, $x(t), C x(t)$ and $(\Phi(C x))(t)$ converge exponentially fast as $t \rightarrow \infty$ and there exists a constant $\gamma>0$ (depending only on $(A, B, C)$ and $\lambda$ ) such that

$$
\|x\|_{L^{\infty}\left(\mathbb{R}_{+}, X\right)}+\|C x\|_{L^{\infty}\left(\mathbb{R}_{+}\right)}+\|\Phi(C x)\|_{L^{\infty}\left(\mathbb{R}_{+}\right)} \leq \gamma\left(\left\|x^{0}\right\|_{1}+\left|\left(\Phi\left(C x^{0}\right)\right)(0)\right|\right) .
$$

Proof. Let $x^{0} \in X_{1}, \alpha \in(\omega(\mathbf{T}), 0)$ and let $g$ denote the impulse response of (43). By assumption, (43) is regular with $B$ or $C$ bounded and with zero feedthrough. Therefore, by Lemma $5.1, g \in L_{\alpha}^{2}\left(\mathbb{R}_{+}\right)$. Consider the integral equation

$$
w=C \mathbf{T} x^{0}-g \star \Phi(w) .
$$

Since $C \mathbf{T} x^{0} \in W_{\text {loc }}^{1,1}\left(\mathbb{R}_{+}\right)$with derivative $\left(C \mathbf{T} x^{0}\right)^{\prime}=C_{\Lambda} \mathbf{T} A x^{0} \in L_{\alpha}^{2}\left(\mathbb{R}_{+}\right)$, it follows from an application of part (b) of Theorem 4.1 with $r_{1}=0$ and $r_{2}=C \mathbf{T} x^{0}$ that (47) has a unique absolutely continuous solution $w$ : $\mathbb{R}_{+} \rightarrow \mathbb{R}$. A routine argument then shows that the function $x: \mathbb{R}_{+} \rightarrow X$ defined by

$$
x(t):=\mathbf{T}_{t} x^{0}-\int_{0}^{t} \mathbf{T}_{t-s} B(\Phi(w))(s) \mathrm{d} s, \quad t \in \mathbb{R}_{+}
$$

is the unique solution of (45). Clearly, if $C$ is bounded, then $x(t) \in \operatorname{dom}(C)=X$ for all $t \in \mathbb{R}_{+}$. If $C$ is unbounded, then, by assumption, $B$ is bounded; using the absolute continuity of $\Phi(w)$ and a standard result on abstract Cauchy problems (see Th. 2.4, p. 107 in Pazy [12]) shows that $x(t) \in \operatorname{dom}(C)=X_{1}$ for all $t \in \mathbb{R}_{+}$. Noting that

$$
C x=C \mathbf{T} x^{0}-G \Phi(w)=C \mathbf{T} x^{0}-g \star \Phi(w)
$$


it follows that $C x=w$. Applying again part (b) of Theorem 4.1 to (47) shows that there exists $\beta \in(\omega(\mathbf{T}), 0)$ such that $\Phi(C x) \in L_{\beta}^{2}\left(\mathbb{R}_{+}\right), C x(t)$ and $(\Phi(C x))(t)$ converge exponentially fast (with convergence rate $\beta$ ) to finite limits as $t \rightarrow \infty$ and, furthermore, there exists $\tilde{\gamma}>0$ (depending only on $(A, B, C)$ and $\lambda$ ) such that

$$
\|C x\|_{L^{\infty}\left(\mathbb{R}_{+}\right)}+\|\Phi(C x)\|_{L^{\infty}\left(\mathbb{R}_{+}\right)}+\left\|(\Phi(C x))^{\prime}\right\|_{L_{\beta}^{2}\left(\mathbb{R}_{+}\right)} \leq \tilde{\gamma}\left(\left\|x^{0}\right\|_{1}+\left|\left(\Phi\left(C x^{0}\right)\right)(0)\right|\right) .
$$

Combining this with part (a) of Lemma 5.2 shows that (46) holds and that

$$
\lim _{t \rightarrow \infty} x(t)=-A^{-1} B \lim _{t \rightarrow \infty}(\Phi(C x))(t),
$$

the convergence being exponential with convergence rate $\beta$.

Remark 5.4. Assume that $\left\{\Phi_{\xi}\right\}_{\xi \in P}$ is an unbiased family of hysteresis operators satisfying, for each $\xi \in P$, (LW), (N1, N2) and (N3) with associated $\lambda>0$ independent of $\xi$. Inequality (46) shows that

$$
\left(\|x\|_{L^{\infty}\left(\mathbb{R}_{+}, X\right)}+\|C x\|_{L^{\infty}\left(\mathbb{R}_{+}\right)}+\left\|\Phi_{\xi}(C x)\right\|_{L^{\infty}\left(\mathbb{R}_{+}\right)}\right) \rightarrow 0 \quad \text { as }\left(\left\|x^{0}\right\|_{1}+\|\xi\|\right) \rightarrow 0
$$

In the infinite-dimensional case, equation (49) is of limited use, since $x^{0}$ is required to approach 0 in the norm of $X_{1}$ rather than $X$. In the finite-dimensional case, however, (49) expresses a Lyapunov-type stability property of the feedback system. Under the extra assumption that there exists a $\mathcal{K}$-function $\kappa$ (that is, $\kappa$ is a continuous non-decreasing function from $\mathbb{R}_{+}$to $\mathbb{R}_{+}$with $\kappa(0)=0$ ) such that

$$
\left|\left(\Phi_{\xi}(u)\right)(0)\right| \leq \kappa(|u(0)|+\|\xi\|), \quad \forall u \in C\left(\mathbb{R}_{+}\right), \forall \xi \in P
$$

it follows from (46) that

$$
\|x\|_{L^{\infty}\left(\mathbb{R}_{+}, X\right)}+\|C x\|_{L^{\infty}\left(\mathbb{R}_{+}\right)}+\left\|\Phi_{\xi}(C x)\right\|_{L^{\infty}\left(\mathbb{R}_{+}\right)} \leq \tilde{\kappa}\left(\left\|x^{0}\right\|_{1}+\|\xi\|\right), \quad \forall x^{0} \in X_{1}, \forall \xi \in P
$$

for a suitable $\mathcal{K}$-function $\tilde{\kappa}$. In the finite-dimensional case this is reminiscent of stability in the large. It follows from (11) that their exist $\mathcal{K}$-functions $\kappa$ such that the backlash, elastic plastic and Prandtl families satisfy (50).

Application of Theorem 4.5. Consider (43) with the feedback law

$$
u=\Phi(v), \quad v^{\prime}=-y
$$

where $\Phi: C\left(\mathbb{R}_{+}\right) \rightarrow C\left(\mathbb{R}_{+}\right)$is a hysteresis operator. The resulting feedback system is then given by

$$
\begin{aligned}
& x^{\prime}=A x+B \Phi(v), \quad x(0)=x^{0} \in X, \\
& v^{\prime}=-C_{\Lambda}\left(x-(z I-A)^{-1} B \Phi(v)\right)-\mathbf{G}(z) \Phi(v), \quad v(0)=v^{0} \in \mathbb{R} .
\end{aligned}
$$

Let $0<\tau \leq \infty$. A solution of $(52)$ on the interval $[0, \tau)$ is a continuous function $(x, v)$ : $[0, \tau) \rightarrow X \times \mathbb{R}$ such that $x(t)-(z I-A)^{-1} B(\Phi(v))(t) \in \operatorname{dom}\left(C_{\Lambda}\right)$ for a.e. $t \in[0, \tau), C_{\Lambda}\left(x-(z I-A)^{-1} B \Phi(v)\right) \in L_{\text {loc }}^{1}([0, \tau))$ and for all $t \in[0, \tau)$

$$
\begin{aligned}
& x(t)=x^{0}+\int_{0}^{t}[A x(s)+B(\Phi(v))(s)] \mathrm{d} s \\
& v(t)=v^{0}-\int_{0}^{t}\left[C_{\Lambda}\left(x(s)-(z I-A)^{-1} B(\Phi(v))(s)\right)+\mathbf{G}(z)(\Phi(v))(s)\right] \mathrm{d} s .
\end{aligned}
$$


Theorem 5.5. Assume that (43) is strongly stable, $0 \in \varrho(A)$ and $\mathbf{G}(0)>0$. Let $\Phi: C\left(\mathbb{R}_{+}\right) \rightarrow C\left(\mathbb{R}_{+}\right)$be a hysteresis operator satisfying (N1-N5) and such that $0 \in \operatorname{clos} \operatorname{NVS} \Phi$. Let $\lambda>0$ be the constant associated with (N3). Assume that there exists $\varepsilon>0$ such that

$$
\operatorname{Re}(\mathbf{G}(i \omega) / i \omega) \geq \varepsilon-1 / \lambda, \quad \text { a.e. } \omega \in \mathbb{R}
$$

Then, for all $\left(x^{0}, v^{0}\right) \in X \times \mathbb{R}$, there exists a unique solution $(x, v)$ of $(52)$ defined on $\mathbb{R}_{+}$such that $x$ and $\Phi(v)$ are bounded, $\lim _{t \rightarrow \infty}\|x(t)\|=0, \lim _{t \rightarrow \infty}(\Phi(v))(t)=0,(\Phi(v))^{\prime} \in L^{2}\left(\mathbb{R}_{+}\right)$and $v^{\prime}+\mathbf{G}(0) \Phi(v) \in L^{2}\left(\mathbb{R}_{+}\right)$. Moreover, there exists $\gamma>0$ (depending only on $(A, B, C), G, \lambda$ and $\varepsilon$ ) such that

$$
\|x\|_{L^{\infty}\left(\mathbb{R}_{+}, X\right)}+\|\Phi(v)\|_{L^{\infty}\left(\mathbb{R}_{+}\right)}+\left\|(\Phi(v))^{\prime}\right\|_{L^{2}\left(\mathbb{R}_{+}\right)} \leq \gamma\left(\left\|x^{0}\right\|+\left|\left(\Phi\left(v^{0}\right)\right)(0)\right|\right)
$$

where $\Phi\left(v^{0}\right)$ denotes the application of $\Phi$ to the constant function $t \mapsto v^{0}$. Furthermore,

(a) if (N6) holds and $0 \in \operatorname{int} \operatorname{NVS} \Phi$, then $v$ is bounded;

(b) if $\lim _{t \rightarrow \infty} C_{\Lambda} \mathbf{T}_{t} x^{0}=0$ and $\lim _{t \rightarrow \infty}(G \theta)(t)$ exists (where $\theta$ denotes the unit-step function), then $\lim _{t \rightarrow \infty} v^{\prime}(t)=0$.

Proof. Let $\left(x^{0}, v^{0}\right) \in X \times \mathbb{R}$. By Lemma 4.4, the integral equation

$$
v(t)=v^{0}-\int_{0}^{t} C_{\Lambda} \mathbf{T}_{s} x^{0} \mathrm{~d} s-\int_{0}^{t}(G \Phi(v))(s) \mathrm{d} s
$$

has a unique absolutely continuous solution $v$ defined on $\mathbb{R}_{+}$. Define the continuous function $x: \mathbb{R}_{+} \rightarrow X$ by

$$
x(t)=\mathbf{T}_{t} x^{0}+\int_{0}^{t} \mathbf{T}_{t-s} B(\Phi(v))(s) \mathrm{d} s .
$$

Then

$$
C_{\Lambda} \mathbf{T} x^{0}+G \Phi(v)=y=C_{\Lambda}\left(x-(z I-A)^{-1} B \Phi(v)\right)+\mathbf{G}(z) \Phi(v)
$$

and a routine argument using standard properties of well-posed systems shows that $(x, v)$ is the unique solution of $(52)$ on $\mathbb{R}_{+}$. Since $0 \in \varrho(A)$, the transfer function $\mathbf{G}(s)$ admits an analytic extension to a neighbourhood of 0 . By assumption, $\mathbf{G}(0)>0$, and it follows that $\mathbf{G}$ satisfies assumption (L). Defining $r \in W_{\text {loc }}^{1,1}\left(\mathbb{R}_{+}\right)$ by $r(t)=v^{0}-\int_{0}^{t} C_{\Lambda} \mathbf{T}_{s} x^{0} \mathrm{~d} s$, then, by strong stability, there exists $\beta>0$ such that

$$
\left\|r^{\prime}\right\|_{L^{2}\left(\mathbb{R}_{+}\right)}=\left\|C_{\Lambda} \mathbf{T} x^{0}\right\|_{L^{2}\left(\mathbb{R}_{+}\right)} \leq \beta\left\|x^{0}\right\|, \quad \forall x^{0} \in X
$$

Therefore we may apply Theorem 4.5 (with $r_{1}=0$ and $r_{2}=r$ ) to (54) to conclude that $\Phi(v)$ is bounded, $\lim _{t \rightarrow \infty}(\Phi(v))(t)=0,(\Phi(v))^{\prime} \in L^{2}\left(\mathbb{R}_{+}\right)$and that there exists $\tilde{\gamma}>0$ (depending only on $(A, B, C), G, \lambda$ and $\varepsilon$ ) such that

$$
\|\Phi(v)\|_{L^{\infty}\left(\mathbb{R}_{+}\right)}+\left\|(\Phi(v))^{\prime}\right\|_{L^{2}\left(\mathbb{R}_{+}\right)} \leq \tilde{\gamma}\left(\left\|x^{0}\right\|+\left|\left(\Phi\left(v^{0}\right)\right)(0)\right|\right)
$$

It follows now from part (b) of Lemma 5.2 that $x$ is bounded, $\lim _{t \rightarrow \infty}\|x(t)\|=0$ and that there exists $\gamma>0$ such that (53) holds. Define the operator $K$ by (27). Since $v^{\prime}=-y=-C_{\Lambda} \mathbf{T} x^{0}-G \Phi(v)$, we obtain, from the fact that $(\Phi(v))^{\prime} \in L^{2}\left(\mathbb{R}_{+}\right)$combined with Lemma 4.3 , that

$$
v^{\prime}=-\mathbf{G}(0) \Phi(v)-K(\Phi(v))^{\prime}-\left(\Phi\left(v^{0}\right)\right)(0)(G \theta-\mathbf{G}(0))-C_{\Lambda} \mathbf{T} x^{0}
$$


and, in particular, $v^{\prime}+\mathbf{G}(0) \Phi(v) \in L^{2}\left(\mathbb{R}_{+}\right)$. Statement (a) follows immediately from Theorem 4.5. To prove statement (b) assume that $\lim _{t \rightarrow \infty} C_{\Lambda} \mathbf{T}_{t} x^{0}=0$ and that $\lim _{t \rightarrow \infty}(G \theta)(t)$ exists. Since $\lim _{t \rightarrow \infty}(\Phi(v))(t)=0$, it follows from (56) that $\lim _{t \rightarrow \infty} v^{\prime}(t)=0$, provided that

$$
\lim _{t \rightarrow \infty}(G \theta)(t)=\mathbf{G}(0) \quad \text { and } \quad \lim _{t \rightarrow \infty}\left(K(\Phi(v))^{\prime}\right)(t)=0 .
$$

The first limit in (57) follows from the facts that $G \theta-\mathbf{G}(0) \in L^{2}\left(\mathbb{R}_{+}\right)$(using part (a) of Lem. 4.3) and that $\lim _{t \rightarrow \infty}((G \theta)(t)-\mathbf{G}(0))$ exists. Finally, by part (b) of Lemma $4.3, K(\Phi(v))^{\prime}=k \star(\Phi(v))^{\prime}$, where $k \in L^{2}\left(\mathbb{R}_{+}\right)$. Since $(\Phi(v))^{\prime} \in L^{2}\left(\mathbb{R}_{+}\right)$, it follows that $\left(k \star(\Phi(v))^{\prime}\right)(t) \rightarrow 0$ as $t \rightarrow \infty$, establishing the second limit in (57).

Finally, whilst Theorems 5.3 and 5.5 might appear vaguely reminiscent of certain (finite-dimensional) results stated (without proof) in Yakubovich [22], we mention that the class of hysteresis nonlinearities considered in [22] is very different from the hysteresis operators in the present paper. In particular, we emphasize that the sector condition imposed on the nonlinearities in [22] is not very natural in a hysteresis context since it excludes backlash and elastic-plastic operators and hence many other hysteresis operators.

\section{Appendix}

Proof of Lemma 2.1. (a) Assume that (LC) is satisfied. Then there exist $\lambda, \delta, \gamma>0$ such that (7) holds. For $\eta \in(0, \gamma]$ and $v \in \mathcal{C}(w ; \delta, \eta)$ we have

$$
\begin{aligned}
\left(\Gamma_{\eta}(v)\right)(t)-w(\alpha)= & r(t)-r(\alpha)-\int_{0}^{t} g(t-s)(\Phi(v))(t) \mathrm{d} s+\int_{0}^{\alpha} g(\alpha-s)(\Phi(w))(s) \mathrm{d} s \\
= & r(t)-r(\alpha)-\int_{0}^{\alpha}(g(t-s)-g(\alpha-s))(\Phi(w))(s) \mathrm{d} s \\
& -\int_{\alpha}^{t} g(t-s)(\Phi(v))(s) \mathrm{d} s \quad \forall t \in[\alpha, \alpha+\eta] .
\end{aligned}
$$

Therefore, for all $\eta \in(0, \gamma]$,

$$
\left|\left(\Gamma_{\eta}(v)\right)(t)-w(\alpha)\right| \leq f(t)+\int_{\alpha}^{t}|g(t-s)|\left|(\Phi(v))(s)-\left(\Phi\left(w_{\gamma}\right)\right)(s)\right| \mathrm{d} s \quad \forall t \in[\alpha, \alpha+\eta],
$$

where $w_{\gamma}$ is defined by (4) and

$$
f(t):=|r(t)-r(\alpha)|+\sup _{s \in[0, \alpha+\gamma]}\left|\left(\Phi\left(w_{\gamma}\right)\right)(s)\right|\left(\int_{0}^{\alpha}|g(t-s)-g(\alpha-s)| \mathrm{d} s+\int_{\alpha}^{t}|g(t-s)| \mathrm{d} s\right) .
$$

Writing $\sigma_{1}(\eta):=\sup _{t \in[\alpha, \alpha+\eta]} f(t)$, then, by continuity of $r$, and since $g \in L_{\text {loc }}^{1}$ and noting that translation is continuous in the $L^{1}$ norm, we see that $\sigma_{1}(\eta) \rightarrow 0$ as $\eta \downarrow 0$. In view of $(7),\left|(\Phi(v))(s)-\left(\Phi\left(w_{\eta}\right)\right)(s)\right| \leq \lambda \delta$ for all $s \in[\alpha, \alpha+\eta]$ and so, for all $\eta \in(0, \gamma]$ and all $t \in[\alpha, \alpha+\eta]$,

$$
\int_{\alpha}^{t}|g(t-s)|\left|(\Phi(v))(s)-\left(\Phi\left(w_{\eta}\right)\right)(s)\right| \mathrm{d} s \leq \lambda \delta \int_{\alpha}^{t}|g(t-s)| \mathrm{d} s=\lambda \delta \int_{0}^{t-\alpha}|g(s)| \mathrm{d} s \leq \delta \sigma_{2}(\eta),
$$

where $\sigma_{2}(\eta):=\int_{0}^{\eta}|g(s)| \mathrm{d} s$. Since $g \in L_{\mathrm{loc}}^{1}\left(\mathbb{R}_{+}\right), \sigma_{2}(\eta) \downarrow 0$ as $\eta \downarrow 0$.

We may now conclude that

$$
\left\|\Gamma_{\eta}(v)-w_{\eta}\right\|_{C([0, \alpha+\eta])}=\sup _{t \in[\alpha, \alpha+\eta]}\left|\left(\Gamma_{\eta}(v)\right)(t)-w(\alpha)\right| \leq \sigma_{1}(\eta)+\delta \sigma_{2}(\eta)=: \sigma(\eta)
$$


Noting that $\sigma(\eta) \leq \delta$ for all $\eta>0$ sufficiently small, it follows that $\Gamma_{\eta}(\mathcal{C}(w ; \delta, \eta)) \subset \mathcal{C}(w ; \delta, \eta)$ for all $\eta>0$ sufficiently small. It remains to prove the strict contraction property. Let $v_{1}, v_{2} \in \mathcal{C}(w ; \delta, \eta)$. Then, again invoking (7),

$$
\begin{aligned}
\left|\left(\Gamma_{\eta}\left(v_{1}\right)\right)(t)-\left(\Gamma_{\eta}\left(v_{2}\right)\right)(t)\right| & =\left|\int_{\alpha}^{t} g(t-s)\left(\left(\Phi\left(v_{1}\right)\right)(s)-\left(\Phi\left(v_{2}\right)\right)(s)\right) \mathrm{d} s\right| \\
& \leq\left(\int_{0}^{\eta}|g(t)| \mathrm{d} t\right) \lambda \sup _{t \in[\alpha, \alpha+\eta]}\left|v_{1}(t)-v_{2}(t)\right| \\
& \leq \lambda \sigma_{2}(\eta)\left\|v_{1}-v_{2}\right\|_{C([0, \alpha+\eta])} \quad \forall t \in[\alpha, \alpha+\eta], \quad \forall \eta \in(0, \gamma] .
\end{aligned}
$$

Therefore,

$$
\begin{aligned}
\left\|\Gamma_{\eta}\left(v_{1}\right)-\Gamma_{\eta}\left(v_{2}\right)\right\|_{C([0, \alpha+\eta])} & =\sup _{t \in[\alpha, \alpha+\eta]}\left|\left(\Gamma_{\eta}\left(v_{1}\right)\right)(t)-\left(\Gamma_{\eta}\left(v_{2}\right)\right)(t)\right| \\
& \leq \lambda \sigma_{2}(\eta)\left\|v_{1}-v_{2}\right\|_{C([0, \alpha+\eta])} \quad \forall \eta \in(0, \gamma]
\end{aligned}
$$

and the strict contraction property follows since $\lambda \sigma_{2}(\eta)<1$ for all $\eta>0$ sufficiently small.

(b) Let $\Phi\left(W_{\text {loc }}^{1,1}\left(\mathbb{R}_{+}\right)\right) \subset W_{\text {loc }}^{1,1}\left(\mathbb{R}_{+}\right), r \in W_{\text {loc }}^{1,1}\left(\mathbb{R}_{+}\right)$and $w \in W^{1,1}([0, \alpha])$. Assume that (LW) is satisfied. Then there exist $\lambda, \delta, \gamma>0$ such that (8) holds. For $\eta \in(0, \gamma]$ and $v \in W(w ; \delta, \eta)$ we have

$$
\left\|\Gamma_{\eta}(v)-w_{\eta}\right\|_{W^{1,1}([0, \alpha+\eta])}=\int_{\alpha}^{\alpha+\eta}\left|\left(\Gamma_{\eta}(v)\right)^{\prime}(t)\right| \mathrm{d} t \leq \sigma_{1}(\eta)+\int_{\alpha}^{\alpha+\eta}\left|\left(g \star\left((\Phi(v))^{\prime}\right)\right)(t)\right| \mathrm{d} t,
$$

where $\sigma_{1}(\eta):=\int_{\alpha}^{\alpha+\eta}\left(\left|r^{\prime}(t)\right|+|(\Phi(w))(0)||g(t)|\right) \mathrm{d} t$. Since $r \in W_{\text {loc }}^{1,1}\left(\mathbb{R}_{+}\right)$and $g \in L_{\text {loc }}^{1}\left(\mathbb{R}_{+}\right)$, we have $\sigma_{1}(\eta) \downarrow 0$ as $\eta \downarrow 0$.

For $\eta \in[0, \gamma]$, define $\tilde{g}, \varphi_{1}, \varphi_{2} \in L^{1}\left(\mathbb{R}_{+}\right)$by

$$
\begin{aligned}
\left.\tilde{g}\right|_{[0, \eta]} & =\left.g\right|_{[0, \eta]}, \quad \tilde{g}(t)=0 \forall t \in \mathbb{R}_{+} \backslash[0, \eta], \\
\left.\varphi_{1}\right|_{[0, \alpha]} & =(\Phi(w))^{\prime}, \quad \varphi_{1}(t)=0 \forall t \in \mathbb{R}_{+} \backslash[0, \alpha], \\
\left.\varphi_{2}\right|_{[\alpha, \alpha+\eta]} & =\left.(\Phi(v))^{\prime}\right|_{[\alpha, \alpha+\eta]}, \quad \varphi_{2}(t)=0 \forall t \in \mathbb{R}_{+} \backslash[\alpha, \alpha+\eta]
\end{aligned}
$$

and note that $(\Phi(v))^{\prime}(t)=\varphi_{1}(t)+\varphi_{2}(t)$ for almost all $t \in[0, \alpha+\eta]$. Since $\Phi(w) \in W^{1,1}([0, \alpha])$, we have $\varphi_{1} \in L^{1}\left(\mathbb{R}_{+}\right)$. Recalling that $g \in L_{\text {loc }}^{1}\left(\mathbb{R}_{+}\right)$, it follows that $g \star \varphi_{1} \in L_{\text {loc }}^{1}\left(\mathbb{R}_{+}\right)$. Therefore, writing

$$
\sigma_{2}(\eta):=\int_{\alpha}^{\alpha+\eta}\left|\left(g \star \varphi_{1}\right)(t)\right| \mathrm{d} t
$$

it is clear that $\sigma_{2}(\eta) \downarrow 0$ as $\eta \downarrow 0$. Moreover,

$$
\begin{aligned}
\int_{\alpha}^{\alpha+\eta}\left|\left(g \star \varphi_{2}\right)(t)\right| \mathrm{d} t & =\int_{\alpha}^{\alpha+\eta}\left|\int_{0}^{t} g(t-s) \varphi_{2}(s) \mathrm{d} s\right| \mathrm{d} t=\int_{\alpha}^{\alpha+\eta}\left|\int_{\alpha}^{t} g(t-s) \varphi_{2}(s) \mathrm{d} s\right| \mathrm{d} t \\
& \leq \int_{0}^{\infty}\left|\left(\tilde{g} \star \varphi_{2}\right)(t)\right| \mathrm{d} t \leq \int_{0}^{\infty}|\tilde{g}(t)| \mathrm{d} t \int_{0}^{\infty}\left|\varphi_{2}(t)\right| \mathrm{d} t \\
& =\int_{0}^{\eta}|g(t)| \mathrm{d} t \int_{\alpha}^{\alpha+\eta}\left|(\Phi(v))^{\prime}(t)\right| \mathrm{d} t .
\end{aligned}
$$


Therefore,

$$
\begin{aligned}
\int_{\alpha}^{\alpha+\eta}\left|\left(g \star\left((\Phi(v))^{\prime}\right)\right)(t)\right| \mathrm{d} t= & \int_{\alpha}^{\alpha+\eta}\left|\left(g \star \varphi_{1}\right)(t)+\left(g \star \varphi_{2}\right)(t)\right| \mathrm{d} t \\
\leq & \sigma_{2}(\eta)+\int_{0}^{\eta}|g(t)| \mathrm{d} t \int_{\alpha}^{\alpha+\eta}\left|\left(\Phi\left(w_{\eta}\right)\right)^{\prime}(t)\right| \mathrm{d} t \\
& +\int_{0}^{\eta}|g(t)| \mathrm{d} t \int_{\alpha}^{\alpha+\eta}\left|(\Phi(v))^{\prime}(t)-\left(\Phi\left(w_{\eta}\right)\right)^{\prime}(t)\right| \mathrm{d} t .
\end{aligned}
$$

By (8)

$$
\int_{\alpha}^{\alpha+\eta}\left|(\Phi(v))^{\prime}(t)-\left(\Phi\left(w_{\eta}\right)\right)^{\prime}(t)\right| \mathrm{d} t \leq \lambda \int_{\alpha}^{\alpha+\eta}\left|v^{\prime}(t)-w_{\eta}^{\prime}(t)\right| \mathrm{d} t=\lambda \int_{\alpha}^{\alpha+\eta}\left|v^{\prime}(t)\right| \mathrm{d} t \leq \lambda \delta .
$$

Writing

$$
\sigma_{4}(\eta):=\int_{0}^{\eta}|g(t)| \mathrm{d} t \quad \text { and } \quad \sigma_{3}(\eta):=\sigma_{4}(\eta) \int_{\alpha}^{\alpha+\eta}\left|\left(\Phi\left(w_{\eta}\right)\right)^{\prime}(t)\right| \mathrm{d} t,
$$

with the properties $\sigma_{3}(\eta), \sigma_{4}(\eta) \downarrow 0$ as $\eta \downarrow 0$, we may now conclude that

$$
\left\|\Gamma_{\eta}(v)-w_{\eta}\right\|_{W^{1,1}([0, \alpha+\eta])}=\sigma_{1}(\eta)+\sigma_{2}(\eta)+\sigma_{3}(\eta)+\lambda \delta \sigma_{4}(\eta)=: \sigma(\eta) \quad \forall \eta \in(0, \gamma] .
$$

Noting that $\sigma(\eta)<\delta$ for all $\eta>0$ sufficiently small, it follows that $\Gamma_{\eta}(W(w ; \delta, \eta)) \subset W(w ; \delta, \eta)$ for all $\eta>0$ sufficiently small. It remains to prove the strict contraction property. Let $v_{1}, v_{2} \in W(w ; \delta, \eta)$. Define $\varphi \in$ $L^{1}\left(\mathbb{R}_{+}\right)$by

$$
\left.\varphi\right|_{[\alpha, \alpha+\eta]}=\left.\left[\left(\Phi\left(v_{1}\right)\right)^{\prime}-\left(\Phi\left(v_{2}\right)\right)^{\prime}\right]\right|_{[\alpha, \alpha+\eta]}, \quad \varphi(t)=0 \forall t \in \mathbb{R}_{+} \backslash[\alpha, \alpha+\eta] .
$$

Then, again using (8)

$$
\begin{aligned}
\left\|\Gamma_{\eta}\left(v_{1}\right)-\Gamma_{\eta}\left(v_{2}\right)\right\|_{W^{1,1}([0, \alpha+\eta])} & =\int_{\alpha}^{\alpha+\eta}\left|\left(\Gamma_{\eta}\left(v_{1}\right)\right)^{\prime}(t)-\left(\Gamma_{\eta}\left(v_{2}\right)\right)^{\prime}(t)\right| \mathrm{d} t \\
& =\int_{\alpha}^{\alpha+\eta}\left|\left(g \star\left[\left(\Phi\left(v_{1}\right)\right)^{\prime}-\left(\Phi\left(v_{2}\right)\right)^{\prime}\right]\right)(t)\right| \mathrm{d} t=\int_{\alpha}^{\alpha+\eta}|(g \star \varphi)(t)| \mathrm{d} t \\
& =\int_{\alpha}^{\alpha+\eta}\left|\int_{0}^{t} g(t-s) \varphi(s) \mathrm{d} s\right| \mathrm{d} t=\int_{\alpha}^{\alpha+\eta}\left|\int_{\alpha}^{t} g(t-s) \varphi(s) \mathrm{d} s\right| \mathrm{d} t \leq \int_{0}^{\infty}|(\tilde{g} \star \varphi)(t)| \mathrm{d} t \\
& \leq \int_{0}^{\infty}|\varphi(t)| \mathrm{d} t \int_{0}^{\infty}|\tilde{g}(t)| \mathrm{d} t=\int_{\alpha}^{\alpha+\eta}\left|\left(\Phi\left(v_{1}\right)\right)^{\prime}(t)-\left(\Phi\left(v_{2}\right)\right)^{\prime}(t)\right| \mathrm{d} t \int_{0}^{\eta}|g(t)| \mathrm{d} t \\
& \leq \lambda \int_{\alpha}^{\alpha+\eta}\left|v_{1}^{\prime}(t)-v_{2}^{\prime}(t)\right| \mathrm{d} t \int_{0}^{\eta}|g(t)| \mathrm{d} t \leq \sigma_{5}(\eta)\left\|v_{1}-v_{2}\right\|_{W^{1,1}([0, \alpha+\eta])} \forall \eta \in(0, \gamma],
\end{aligned}
$$

where $\sigma_{5}(\eta):=\lambda \int_{0}^{\eta}|g(t)| \mathrm{d} t<1$ for all $\eta>0$ sufficiently small.

Proof of Lemma 4.3. (a) Note that the Laplace transform of $G \theta-\mathbf{G}(0)$ is the function $s \mapsto(\mathbf{G}(s)-\mathbf{G}(0)) / s$, which by assumption $(\mathrm{L})$ is in $H^{2}\left(\mathbb{C}_{0}\right)$. Invoking a well-known result of Paley and Wiener shows that $G \theta-\mathbf{G}(0)$ $\in L^{2}\left(\mathbb{R}_{+}\right)$.

(b) The transfer function of $K$ is $\mathbf{K}(s)=(\mathbf{G}(s)-\mathbf{G}(0)) / s$, and so, by assumption $(\mathrm{L}), \mathbf{K} \in H^{2}\left(\mathbb{C}_{0}\right) \cap H^{\infty}\left(\mathbb{C}_{0}\right)$. Thus, $K$ is a convolution operator with kernel $k \in L^{2}\left(\mathbb{R}_{+}\right)$and $K \in \mathcal{L}\left(L^{2}\left(\mathbb{R}_{+}\right)\right)$. 
(c) Equation (28) follows from a straightforward calculation. By statements (a) and (b), $G \theta-\mathbf{G}(0)$ and $K u^{\prime}$ are in $L^{2}\left(\mathbb{R}_{+}\right)$and therefore we obtain from $(28)$ that $G u-\mathbf{G}(0) u \in L^{2}\left(\mathbb{R}_{+}\right)$.

Proof of Lemma 5.2. Let $x^{0} \in X, u \in W_{\text {loc }}^{1,2}\left(\mathbb{R}_{+}\right)$and assume that $0 \in \varrho(A)$. The solution of (43a) can be written in the form

$$
x(t)=\mathbf{T}(t) x^{0}+(F u)(t), \quad \forall t \in \mathbb{R}_{+},
$$

where $F: L_{\text {loc }}^{2}\left(\mathbb{R}_{+}\right) \rightarrow L_{\text {loc }}^{2}\left(\mathbb{R}_{+}, X\right)$ is the shift-invariant operator defined by

$$
(F v)(t)=\int_{0}^{t} \mathbf{T}_{t-s} B v(s) \mathrm{d} s, \quad v \in L_{\mathrm{loc}}^{2}\left(\mathbb{R}_{+}\right) .
$$

Since $F$ commutes with integration (by shift-invariance), it follows from (59) that

$$
x(t)+A^{-1} B u(t)=\mathbf{T}(t) x^{0}+\left(K u^{\prime}\right)(t)+u(0) F \theta+A^{-1} B u(0), \quad \forall t \in \mathbb{R}_{+},
$$

where $K: L_{\text {loc }}^{2}\left(\mathbb{R}_{+}\right) \rightarrow L_{\text {loc }}^{2}\left(\mathbb{R}_{+}, X\right)$ is the shift-invariant operator defined by

$$
(K v)(t)=\int_{0}^{t}\left((F v)(s)+A^{-1} B v(s)\right) \mathrm{d} s, \quad v \in L_{\mathrm{loc}}^{2}\left(\mathbb{R}_{+}\right) .
$$

A straightforward calculation shows that $F \theta=(\mathbf{T}-I) A^{-1} B$ and

$$
(K v)(t)=\int_{0}^{t} \mathbf{T}_{t-s} A^{-1} B v(s) \mathrm{d} s, \quad v \in L_{\mathrm{loc}}^{2}\left(\mathbb{R}_{+}\right) .
$$

Hence,

$$
x(t)+A^{-1} B u(t)=\mathbf{T}(t)\left(x^{0}+A^{-1} B u(0)\right)+\int_{0}^{t} \mathbf{T}_{t-s} A^{-1} B u^{\prime}(s) \mathrm{d} s, \quad \forall t \in \mathbb{R}_{+} .
$$

(a) Assume that $\omega(\mathbf{T})<0$ and $u^{\prime} \in L_{\beta}^{2}\left(\mathbb{R}_{+}\right)$for some $\beta \in(\omega(\mathbf{T}), 0)$. It follows from (60) and the fact that the weighted semigroup $t \mapsto \mathrm{e}^{-\beta t} \mathbf{T}_{t}$ is exponentially stable that there exists $\gamma>0$ such that the inequality in statement (a) holds.

(b) Assume that $\mathbf{T}$ is strongly stable, $B$ is infinite-time admissible and $u^{\prime} \in L^{2}\left(\mathbb{R}_{+}\right)$. Using (60) combined with the strong stability of $\mathbf{T}$ and the infinite-time admissibility of $B$, shows that there exists $\gamma>0$ such that the inequality in statement (b) holds. To prove that $\lim _{t \rightarrow \infty}\left\|x(t)+A^{-1} B u(t)\right\|=0$, note that by (60) and the strong stability of $\mathbf{T}$ it is sufficient to show that

$$
z(t):=\int_{0}^{t} \mathbf{T}_{t-s} A^{-1} B u^{\prime}(s) \mathrm{d} s \rightarrow 0, \quad \text { as } t \rightarrow \infty .
$$

For $\tau \geq 0$ we have

$$
z(t)=\mathbf{T}_{t-\tau} z(\tau)+A^{-1} \int_{\tau}^{t} \mathbf{T}_{t-s} B u^{\prime}(s) \mathrm{d} s, \quad \forall t \geq \tau .
$$

By infinite-time admissibility there exists $\alpha>0$ such that

$$
\left\|A^{-1} \int_{t_{0}}^{t} \mathbf{T}_{t-s} B u^{\prime}(s) \mathrm{d} s\right\| \leq \alpha\left(\int_{t_{0}}^{t}\left|u^{\prime}(s)\right|^{2} \mathrm{~d} s\right)^{1 / 2}, \quad \forall t \geq t_{0} \geq 0 .
$$


Let $\varepsilon>0$. Since $u^{\prime} \in L^{2}\left(\mathbb{R}_{+}\right)$, we obtain from (63) that there exists $\tau \geq 0$ such that

$$
\left\|A^{-1} \int_{\tau}^{t} \mathbf{T}_{t-s} B u^{\prime}(s) \mathrm{d} s\right\| \leq \varepsilon / 2, \quad \forall t \geq \tau .
$$

Finally, by strong stability, there exists $\sigma \geq 0$ such that

$$
\left\|\mathbf{T}_{t} z(\tau)\right\| \leq \varepsilon / 2, \quad \forall t \geq \sigma .
$$

It follows from (62) that $\|z(t)\| \leq \varepsilon$ for all $t \geq \sigma+\tau$.

\section{REFERENCES}

[1] M. Brokate, Hysteresis operators, in Phase Transitions and Hysteresis, edited by A. Visintin. Springer-Verlag, Berlin (1994) $1-38$.

[2] M. Brokate and J. Sprekels, Hysteresis and Phase Transitions. Springer-Verlag, New York (1996).

[3] C. Corduneanu, Almost Periodic Functions, 2nd Edition. Chelsea Publishing Company, New York (1989).

[4] R.F. Curtain, H. Logemann and O. Staffans, Stability results of Popov-type for infinite-dimensional systems with applications to integral control, Mathematics Preprint 01/09. University of Bath (2001). Proc. London Math. Soc. (to appear). Available at http://www.maths.bath.ac.uk/MATHEMATICS/preprints.html

[5] R.F. Curtain and G. Weiss, Well-posedness of triples of operators in the sense of linear systems theory, in Control and Estimation of Distributed Parameter System, edited by F. Kappel, K. Kunisch and W. Schappacher. Birkhäuser Verlag, Basel (1989) 41-59.

[6] G. Gripenberg, S.-O. Londen and O.J. Staffans, Volterra Integral and Functional Equations. Cambridge University Press, Cambridge (1990).

[7] W. Hahn, Stability of Motion. Springer-Verlag, Berlin (1967).

[8] M.A. Krasnosel'skiǔ and A.V. Pokrovskiǔ. Systems with Hysteresis. Springer-Verlag, Berlin (1989).

[9] H. Logemann and A.D. Mawby, Low-gain integral control of infinite-dimensional regular linear systems subject to input hysteresis, in Advances in Mathematical Systems Theory, edited by F. Colonius et al. Birkhäuser, Boston (2001) 255-293.

[10] H. Logemann and E.P. Ryan, Time-varying and adaptive integral control of infinite-dimensional regular linear systems with input nonlinearities. SIAM J. Control Optim. 38 (2000) 1120-1144.

[11] J.W. Macki, P. Nistri and P. Zecca, Mathematical models for hysteresis. SIAM Rev. 35 (1993) 94-123.

[12] A. Pazy, Semigroups of Linear Operators and Applications to Partial Differential Equations. Springer-Verlag, New York (1983).

[13] D. Salamon, Realization theory in Hilbert space. Math. Systems Theory 21 (1989) 147-164.

[14] D. Salamon, Infinite-dimensional linear systems with unbounded control and observation: A functional analytic approach. Trans. Amer. Math. Soc. 300 (1987) 383-431.

[15] O.J. Staffans, Well-Posed Linear Systems. Book manuscript (in preparation). Available at http://www.abo.fi/ staffans/

[16] O.J. Staffans, J-energy preserving well-posed linear systems. Int. J. Appl. Math. Comput. Sci. 11 (2001) 1361-1378.

[17] O.J. Staffans, Quadratic optimal control of stable well-posed linear systems. Trans. Amer. Math. Soc. 349 (1997) $3679-3715$.

[18] O.J. Staffans and G. Weiss, Transfer functions of regular linear systems, Part II: The system operator and the Lax-Phillips semigroup. Trans. Amer. Math. Soc. 354 (2002) 3229-3262.

[19] M. Vidyasagar, Nonlinear Systems Analysis, 2nd Edition. Prentice Hall, Englewood Cliffs, NJ (1993).

[20] G. Weiss, Transfer functions of regular linear systems, Part I: Characterization of regularity. Trans. Amer. Math. Soc. 342 (1994) 827-854.

[21] G. Weiss, The representation of regular linear systems on Hilbert spaces, in Control and Estimation of Distributed Parameter System, edited by F. Kappel, K. Kunisch and W. Schappacher. Birkhäuser Verlag, Basel (1989) 401-416.

[22] V.A. Yakubovich, The conditions for absolute stability of a control system with a hysteresis-type nonlinearity. Soviet Phys. Dokl. 8 (1963) 235-237 (translated from Dokl. Akad. Nauk SSSR 149 (1963) 288-291). 